\title{
Two New Types of Multiple Granulation Rough Set
}

\author{
Weihua Xu, ${ }^{1,2}$ Xiaoyan Zhang, ${ }^{1}$ and Wenxiu Zhang ${ }^{3}$ \\ ${ }^{1}$ School of Mathematics and Statistics, Chongqing University of Technology, Chongqing 400054, China \\ ${ }^{2}$ School of Management, Xian Jiaotong University, Xian, Shaanxi 710049, China \\ ${ }^{3}$ School of Science, Xian Jiaotong University, Xian, Shaanxi 710019, China
}

Correspondence should be addressed to Xiaoyan Zhang; zhangxyms@gmai.com

Received 6 November 2012; Accepted 22 November 2012

Academic Editors: A. Bellouquid and T. Y. Kam

Copyright (C) 2013 Weihua Xu et al. This is an open access article distributed under the Creative Commons Attribution License, which permits unrestricted use, distribution, and reproduction in any medium, provided the original work is properly cited.

\begin{abstract}
In the paper proposed two new types of the multiple granulation rough set models, where a target concept is approximated from two different kinds of views by using the equivalence classes induced by multiple granulations. A number of important properties of the two types of MGRS are investigated. From the properties, it can be found that Pawlak's and Qian's rough set models are special instances of those of our MGRS. Moreover, several important measures are presented in two types of MGRS, such as rough measure and quality of approximation. Furthermore, the relationship and difference are discussed carefully among Pawlak's rough set, Qian's MGRS, and two new types of MGRS. In order to illustrate our multiple granulations rough set model, some examples are considered, which are helpful for applying this theory in practical issues. One can get that the paper is meaningful both in the theory and in application for the issue of knowledge reduction in complex information systems.
\end{abstract}

\section{Introduction}

Rough set theory proposed by Pawlak [1-3] is an extension of the classical set theory and can be regarded as a soft computing tool to handle imprecision, vagueness, and uncertainty in data analysis. The theory has found its successive applications in the fields of pattern recognition [4], medical diagnosis [5], data mining [6-8], conflict analysis [9], algebra [10-12], and so on. Recently, the theory has generated a great deal of interest among more and more researchers.

The classical rough set theory is based upon the classification mechanism, from which the classification can be viewed as an equivalence relation, and knowledge granule induced by the equivalence relation can be viewed as a partition of the universe of discourse. In rough set theory, two classical sets, so-called lower and upper approximations or Pawlak's rough approximations, a constructed and any subset of a universe of discourse can be expressed by them. In framework based on rough set theory, an attribute set is viewed as a granular space, which partitions the universe into some knowledge granules or elemental concepts. Partition, granulation, and approximation are the methods widely used in human reasoning $[13,14]$. Rough set methodology presents a novel paradigm to deal with uncertainty and has been applied to feature selection [15], knowledge reduction [1619], rule extraction [20-23], uncertainty reasoning [24, 25], and granular computing [26-31].

In practice, due to the existence of uncertainty and complexity of particular problem, the problem would not be settled perfectly by means of classical rough set. Therefore, it is vital to generalize the classical rough set model. To overcome this limitation, classical rough sets have been accomplished to several interesting and meaningful general models in recent years, which include rough set model based on tolerance relations [32], rough set model based on neighborhood operators [33], Bayesian rough set model [34], fuzzy rough set model [35], rough fuzzy set model [35], and fuzzy probabilistic rough set model [3, 9, 19, 36-45].

On the other hand, information granules have played a significant role in human cognitive processes. Information granules refer to pieces, classes, and groups divided in 
accordance with characteristics and performances of complex information in the process of human understanding, reasoning, and decision making. Such information processing is called the information granulation. Zadeh firstly proposed and discussed the issue of fuzzy information granulation [46] in 1979. Then, the basic idea of information granulation has been applied to many fields, such as theory of rough sets [1, 2, 47], fuzzy sets [14, 48], and evidence theories [49], and a growing number of scholars are concerned about the discipline. In 1985, Hobbs proposed the concept of granularity [50]. Zadeh firstly presented the concept of granular computing [46] in the period from 1996 to 1997. At this time, granular computing has played a more and more important role in soft computing, knowledge discovery, and data mining, and ones have achieved a large amount of excellent results $[19,27,30,51-58]$.

However, in essence, the approximations in the past approaches are still based on a singleton granulation induced from an indiscernibility relation, which can be applied to knowledge representation in distributive systems and groups of intelligent agents. In view of granular computing, an equivalence relation on the universe can be regarded as a granulation, and a partition on the universe can be regarded as a granulation space $[56,59]$. Hence, the classical rough set theory is based on a single granulation (only one equivalence relation). Note that any attribute set can induce a certain equivalence relation in an information system. In the literature, to more widely apply the rough set theory in practical applications, Qian et al. [60] extended Pawlak's single-granulation rough set model to a multi granulation rough set model (MGRS), where the set approximations are defined by multiple equivalence relations on the universe.

The main objective of this paper is to extend Pawlak's single-granulation rough set model and Qian's multigranulation rough set model (MGRS) to two new types of multiple granulation rough set model, where the set approximations are defined by using multiple equivalence relations on the universe. The rest of the paper is organized as follows. Some preliminary concepts in Pawlak's rough set theory and main concepts in Qian's MGRS are briefly reviewed in Section 2. In Sections 3 and 4, for an information system, based on multiple equivalence relations, two new types of multiple granulation rough set model are obtained, respectively, where a target concept is approximated from two different kinds of views by using the equivalence classes induced by multiple granulations. And a number of important properties of the two types of MGRS are investigated. It is shown that some of the properties of Pawlak's and Qian's rough set theory are special instances of those of our MGRS. Several important measures are presented in two types of MGRS, such as rough measure, quality of approximation. In Section 5, the relationship and difference are discussed among Pawlak's rough set, Qian's MGRS, and two new types of MGRS. Furthermore, to illustrate our multiple granulations rough set model, some examples are presented, which are helpful for applying this theory in practical issues. And finally, the paper is concluded by a summary and outlook for further research in Section 6.

\section{Preliminaries}

The following recalls necessary concepts and preliminaries required in the sequel of our work. Detailed description of the theory can be found in the literatures $[1-3,19,60]$.

2.1. Pawlak's Rough Set. The notion of information system (sometimes called data tables, attribute-value systems, knowledge representation systems, etc.) provides a convenient tool for the representation of objects in terms of their attribute values.

An information system is an ordered quadruple $\mathscr{I}=$ $(U, A T, V, f)$, where

(i) $U=\left\{u_{1}, u_{2}, \ldots, u_{n}\right\}$ is a nonempty finite set of objects,

(ii) $A T=\left\{a_{1}, a_{2}, \ldots, a_{p}\right\}$ is a nonempty finite set of attributes,

(iii) $V=\bigcup_{a \in A T} V_{a}$ and $V_{a}$ is a domain of attribute $a$,

(iv) $f: U \times A T \rightarrow V$ is a function such that $f(u, a) \in$ $V_{a}$, for every $a \in A T, x \in U$, called an information function.

An information system with the decision is a special case of information systems in which, among the attributes, we distinguish the ones called decision attribute. The other attributes are called condition attributes. Therefore, $\mathscr{I}=$ $(U, C \cup\{d\}, V, f)$ and $C \cap\{d\}=\phi$, where sets $C$ and $\{d\}$ are the condition attributes and the decision attribute, respectively.

Let $\mathscr{I}=(U, A T, V, f)$ be an information system, for $A \subseteq$ At, and denote

$$
R_{A}=\{(u, v) \in U \times U \mid f(u, a)=f(v, a), \forall a \in A\},
$$

then $R_{A}$ is reflexive, symmetric, and transitive. So it is an equivalence relation on $U$.

Moreover, denote

$$
\begin{aligned}
{[u]_{A} } & =\left\{v \in U \mid(u, v) \in R_{A}\right\}, \\
\frac{U}{A} & =\left\{[u]_{A}, \forall u \in U\right\},
\end{aligned}
$$

then $[u]_{A}$ is called the equivalence class of $u$, and $U / A$ is called the equivalence class set of $U$. For equivalence classes and equivalence relations, the following properties hold.

(i) If $B \subseteq A$, then $R_{A} \subseteq R_{B}$.

(ii) If $B \subseteq A$, then $[u]_{A} \subseteq[u]_{B}$.

(iii) If $v \in[u]_{A}$, then $[u]_{A}=[v]_{A}$.

For any subset $X \subseteq U$ and $A \subseteq A T$ in information system $\mathscr{I}=(U, A T, V, f)$, the Pawlak's lower and upper approximations of $X$ with respect to equivalence relation $R_{A}$ could be defined as follows (see $[1-3,19])$ :

$$
\begin{gathered}
\underline{R_{A}}(X)=\left\{u \in U \mid[u]_{A} \subseteq X\right\}, \\
\overline{R_{A}}(X)=\left\{u \in U \mid[u]_{A} \cap X \neq \emptyset\right\} .
\end{gathered}
$$

The area of uncertainty or boundary is defined as

$$
\operatorname{Bnd}_{A}(X)=\overline{R_{A}}(X)-\underline{R_{A}}(X) .
$$


To measure the imprecision and roughness of a rough set, Pawlak recommended that $X \neq \emptyset$ has the ratio

$$
\rho_{A}(X)=1-\frac{\left|\underline{R_{A}}(X)\right|}{\left|\overline{R_{A}}(X)\right|},
$$

which is called the rough measure of $X$ by equivalence $R_{A}$.

Furthermore, for an information system with the decision $\mathscr{I}=(U, C \cup\{d\}, V, f)$ and $A \subseteq C$, a frequently applied measure for the situation is the quality of approximation of $R_{d}$ by $R_{A}$, also called the degree of dependency. It is defined as

$$
\gamma(A, d)=\frac{1}{|U|} \sum_{j=1}^{k} \underline{R_{A}}\left(D_{j}\right),
$$

where $R_{d}=\{(u, v) \in U \times U \quad \mid g(u, d)=g(v, d)\}$, and $U / d=$ $\left\{[u]_{d}\right.$, for all $\left.u \in U\right\}=\left\{D_{1}, D_{2}, \ldots, D_{k}\right\}$.

2.2. Qian's Multiple Granulation Rough Set. In the rough set model MGRS, unlike Pawlak's rough set theory, a target concept is approximated through multiple partitions induced by multiple equivalence relations [60]. In the following, we recall the relevant concepts about MGRS, and the description in detail can be found in [60].

Suppose that $\mathscr{I}=(U, A T, V, f)$ is an information system, and $P_{1}, P_{2}, \ldots, P_{m}$ are $m$-attribute subsets. A lower approximation and an upper approximation of $X$ are related to $P_{1}, P_{2}, \ldots, P_{m}$ by the following:

$$
\begin{gathered}
\underline{\sum_{i=1}^{m} P_{i} X=\bigcup\left\{u \mid[u]_{P_{i}} \subseteq X, \text { for some } i \leq m\right\},} \\
\overline{\sum_{i=1}^{m} P_{i} X}=\sim \sum_{i=1}^{m} P_{i}(\sim X) .
\end{gathered}
$$

Similarly, the boundary region in MGRS can be expressed as

$$
\operatorname{Bnd}_{\sum_{i=1}^{m} P_{i}}(X)=\overline{\sum_{i=1}^{m} P_{i}} X \backslash \sum_{i=1}^{m} P_{i} X .
$$

Figure 1 shows the difference between Pawlak's rough set model and the MGRS model.

From the figure, we can find that the bias region is the lower approximation of a set $X$ obtained by a single granulation $P \cup Q$, which are expressed by the equivalence classed in the quotient set $U /(P \cup Q)$, and the shadow region is the lower approximation of $X$ induced by two granulations $P+Q$, which are characterized by the equivalence classes in quotient set $U / P$ and the quotient set $U / Q$.

\section{The First Type of Multiple Granulation Rough Set}

In this section, we will propose the first type of multiple granulation rough set.

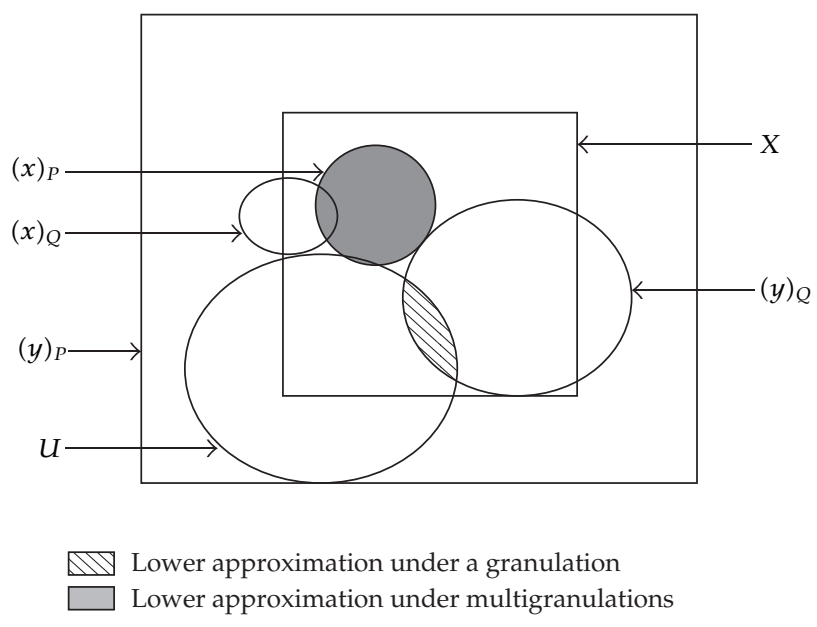

FIGURE 1: Difference between Pawlak's rough set model and MGRS.

3.1. The First Type of Two Granulation Rough Set. We first discuss the first type of two granulation approximations of a target set by using two equivalence relations in an information system.

Definition 1. Let $\mathscr{I}=(U, A T, V, f)$ be an information system and $B, A \subseteq A T$. The operators $F R_{A+B}$ and $\overline{F R_{A+B}}: \mathscr{P}(U) \rightarrow$ $\mathscr{P}(U)$ are defined as follows. For all $X \in \mathscr{P}(U)$,

$$
\begin{gathered}
\frac{F R_{A+B}}{}(X)=\left\{u \mid[u]_{A} \subseteq X \text { or }[u]_{B} \subseteq X\right\}, \\
\overline{F R_{A+B}}(X)=\left\{u \mid[u]_{A} \cap X \neq \emptyset,[u]_{B} \cap X \neq \emptyset\right\} .
\end{gathered}
$$

We call them the first type of two granulation lower and upper approximation operators, and we call $\mathrm{FR}_{A+B}(X)$ and $\overline{F R_{A+B}}(X)$ the first type of two granulation lower approximation set and upper approximation of $X$, respectively.

Moreover, if $F R_{A+B}(X) \neq \overline{F R_{A+B}}(X)$, we say that $X$ is the first type of rough set with respect to the two granulation spaces $A$ and $B$. Otherwise, we say that $X$ is the first type of a definable set with respect to the two granulation spaces $A$ and $B$.

The area of uncertainty or boundary region of this rough set is defined as

$$
\operatorname{Bnd}_{R_{A+B}}^{F}(X)=\overline{F R_{A+B}}(X)-\underline{F R_{A+B}}(X) .
$$

It can be found that the first two granulation rough set will be Pawlak's rough set when two granulation spaces $A$ and $B$ satisfy $A=B$. To describe conveniently in our context, we express the first type of two granulation rough set by using the 1st TGRS.

In Qian's MGRS, the upper approximation set is characterized by the complementary set of the lower approximation of the complementary target concept $X$, which is not consistent with Pawlak's rough set. However, by the previous definition, it can be seen that the 1st TGRS lower and upper approximations are consistent with Pawlak's rough set. Furthermore, ones can find that the 1st TGRS lower and upper approximations are defined through using the 


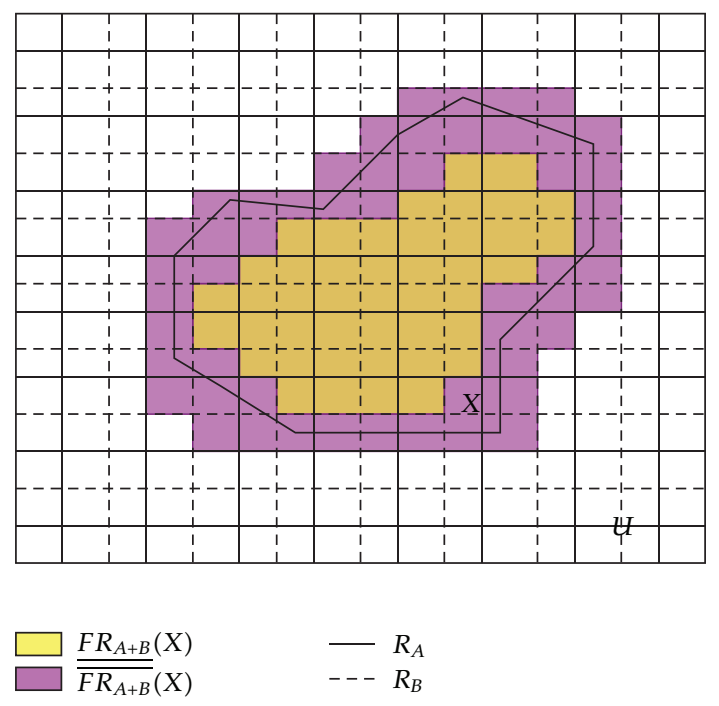

FIGURE 2: The 1st TGRS in an information system.

equivalence classes induced by multiequivalence relations in an information system, whereas Pawlak's lower and upper approximations are represented via those derived by only on equivalence relation.

One can understand the first two granulation rough set and show the difference between the Qian's MGRS and Pawlak's rough set through Figure 2.

Just from Definition 1, we can obtain the following properties in the 1st TGRS in an information system.

Proposition 2. Let $\mathscr{I}=(U, A T, V, f)$ be an information system, $B, A \subseteq A T$ and $X \subseteq U$. Then the following properties hold.

$$
\begin{aligned}
& \left(\mathrm{FL}_{1}\right) \overline{F R_{A+B}}(X) \subseteq X \quad(\text { Contraction), } \\
& \left(\mathrm{FU}_{1}\right) \overline{F R_{A+B}}(X) \supseteq X \quad(\text { Extension }), \\
& \left(\mathrm{FL}_{2}\right) \overline{F R_{A+B}}(\sim X)=\sim \overline{F R_{A+B}}(X) \quad(\text { Duality), } \\
& \left(\mathrm{FU}_{2}\right) \overline{F R_{A+B}}(\sim X)=\sim \overline{F R_{A+B}}(X) \quad(\text { Duality), } \\
& \left(\mathrm{FL}_{3}\right) \overline{F R_{A+B}}(\emptyset)=\emptyset \quad(\text { Normality), } \\
& \left(\mathrm{FU}_{3}\right) \overline{F R_{A+B}}(\emptyset)=\emptyset \quad(\text { Normality), } \\
& \left(\mathrm{FL}_{4}\right) \overline{F R_{A+B}}(U)=U \quad(\text { Conormality), } \\
& \left(\mathrm{FU}_{4}\right) \overline{\overline{F R_{A+B}}}(U)=U \quad(\text { Conormality), } \\
& \left(\mathrm{FL}_{5}\right) \overline{F R_{A+B}}(X)=\overline{F R_{B+A}}(X) \quad \text { (Commutativity), } \\
& \left(\mathrm{FU}_{5}\right) \overline{F R_{A+B}}(X)=\overline{F R_{B+A}}(X) \quad \text { (Commutativity). }
\end{aligned}
$$

Proof. It is obvious that all terms hold when $A=B$. When $A \neq B$, the proposition can be proved as follows.

$\left(\mathrm{FL}_{1}\right)$ For any $u \in F R_{A+B}(X)$, it can be known that $[u]_{A} \subseteq$ $X$ or $[u]_{B} \subseteq X$ by Definition 1. However, $u \in[u]_{A}$ and $u \in$ $[u]_{B}$. So we can have $u \in X$. Hence, $F R_{A+B}(X) \subseteq X$.
$\left(\mathrm{FU}_{1}\right)$ For any $u \in X$, we have $u \in[u]_{A}$ and $u \in[u]_{B}$. So $[u]_{A} \cap X \neq \emptyset$ and $[u]_{B} \cap X \neq \emptyset$, that is to say, $u \in \overline{F R_{A+B}}(X)$. Hence, $X \subseteq \overline{F R_{A+B}}(X)$.

$\left(\mathrm{FL}_{2}\right)$ For any $u \in F R_{A+B}(\sim X)$, then

$$
\begin{aligned}
u \in \underline{F R_{A+B}}(\sim X) & \Longleftrightarrow[u]_{A} \subseteq \sim X, \text { or }[u]_{B} \subseteq \sim X, \\
& \Longleftrightarrow[u]_{A} \cap X=\emptyset, \text { or }[u]_{B} \cap X=\emptyset, \\
& \Longleftrightarrow u \notin \overline{F R_{A+B}}(X), \\
& \Longleftrightarrow u \in \sim \overline{F R_{A+B}}(X) .
\end{aligned}
$$

Hence, $F R_{A+B}(\sim X)=\sim \overline{F R_{A+B}}(X)$.

$\left(\mathrm{FU}_{2}\right)$ By $\left(\mathrm{FL}_{2}\right)$, we have $F R_{A+B}(X)=\sim \overline{F R_{A+B}}(\sim X)$. So it can be obtain that $\sim F R_{A+B}\left(\overline{X)=\bar{F} R_{A+B}}(\sim X)\right.$.

$\left(\mathrm{FL}_{3}\right)$ From $\left(\mathrm{FL}_{1}\right) \overline{\text {, we have }} F R_{A+B}(\emptyset) \subseteq \emptyset$. besides, it is well known that $\emptyset \subseteq \overline{F R}_{A+B}(\emptyset)$. So, $F R_{A+B}(\emptyset)=\emptyset$.

$\left(\mathrm{FU}_{3}\right)$ If $\overline{F R_{A+B}}(\emptyset) \neq \emptyset$, then there must exist a $u \in$ $\overline{F R_{A+B}}(\emptyset)$. So we can find that $[u]_{A} \cap \emptyset \neq \emptyset$ and $[u]_{B} \cap \emptyset \neq \emptyset$. Obviously, this is a contradiction. Thus, $\overline{F R_{A+B}}(\emptyset)=\emptyset$.

$\left(\mathrm{FL}_{4}\right) F R_{A+B}(U)=F R_{A+B}(\sim \emptyset)=\sim \overline{F R_{A+B}}(\emptyset)=\sim \emptyset=U$.
$\left(\mathrm{FU}_{4}\right) \overline{\overline{F R_{A+B}}}(U)=\overline{\overline{F R_{A+B}}}(\sim \emptyset)=\sim F R_{A+B}(\emptyset)=\sim \emptyset=U$.
$\left(\mathrm{FL}_{5}\right)$ and $\left(\mathrm{FU}_{5}\right)$ can be proved directly by Definition 1.

In order to discover the relationship between the 1st TGRS approximations of a single set and the 1st TGRS approximations of two sets described on the universe, the following properties are given.

Proposition 3. Let $\mathscr{I}=(U, A T, V, f)$ be an information system, $B, A \subseteq A T$ and $X, Y \subseteq U$. Then the following properties hold.

$$
\begin{aligned}
& \left(\mathrm{FL}_{6}\right) \frac{F R_{A+B}(X \cap Y) \subseteq \underline{F R_{A+B}}(X) \cap \underline{F R_{A+B}}(Y) \quad(L-}{\text { Multiplication), }} \\
& \left(\mathrm{FU}_{6}\right) \overline{F R_{A+B}}(X \cup Y) \supseteq \overline{F R_{A+B}}(X) \cup \overline{F R_{A+B}}(Y) \quad(L- \\
& \text { Addition), } \\
& \left(\mathrm{FL}_{7}\right) X \subseteq Y \Rightarrow \underline{F R_{A+B}}(X) \subseteq \overline{F R_{A+B}}(Y) \quad \text { (Granularity), } \\
& \left(\mathrm{FU}_{7}\right) X \subseteq Y \Rightarrow \overline{F R_{A+B}}(X) \subseteq \overline{F R_{A+B}}(Y) \quad \text { (Granularity), } \\
& \left(\mathrm{FL}_{8}\right) \frac{F R_{A+B}(X \cup Y)}{\overline{\text { Addition }),}} \\
& \left(\mathrm{FU}_{8}\right) \overline{F R_{A+B}}(X) \cup \overline{F R_{A+B}}(Y) \quad(U- \\
& \\
& \text { Multiplication). }
\end{aligned}
$$

Proof. It is obvious that all terms hold when $A=B$ or $X=$ $Y$. When $A \neq B$ and $X \neq Y$, the proposition can be proved as follows.

$\left(\mathrm{FL}_{6}\right)$ For any $u \in F R_{A+B}(X \cap Y)$, we have that $[u]_{A} \subseteq$

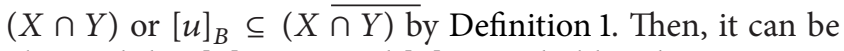
obtained that $[u]_{A} \subseteq X$ and $[u]_{A} \subseteq Y$ hold at the same time or $[u]_{B} \subseteq X$ and $[u]_{B} \subseteq Y$ hold at the same time. So, not only $[u]_{A} \subseteq X$ or $[u]_{B} \subseteq X$ hold, but $[u]_{A} \subseteq Y$ or $[u]_{B} \subseteq Y$ 
hold at the same time. That is to say that $u \in F R_{A+B}(X)$ and $u \in F R_{A+B}(Y)$, that is, $u \in F R_{A+B}(X) \cap F R_{A+B} \overline{(Y)}$.

Hence, $F R_{A+B}(X \cap Y) \overline{\subseteq F R_{A+B}}(X) \overline{\cap F R_{A+B}}(Y)$.

$\left(\mathrm{FU}_{6}\right)$ For any $u \in \overline{F R_{A+B}}(X) \cup \overline{F R_{A+B}}(Y)$, we have $u \in$ $\overline{F R_{A+B}}(X)$ or $u \in \overline{F R_{A+B}}(Y)$. Then $[u]_{A} \cap X \neq \emptyset$ and $[u]_{B} \cap$ $X \neq \emptyset$ hold at the same time or $[u]_{A} \cap Y \neq \emptyset$ and $[u]_{B} \cap Y \neq \emptyset$ hold at the same time. So, not only $[u]_{A} \cap(X \cup Y) \neq \emptyset$ hold, but $[u]_{B} \cap(X \cup Y) \neq \emptyset$ hold. That is to say $u \in \overline{F R_{A+B}}(X \cup Y)$.

Hence, $\overline{F R_{A+B}}(X \cap Y) \supseteq \overline{F R_{A+B}}(X) \cup \overline{F R_{A+B}}(Y)$.

$\left(\mathrm{FL}_{7}\right)$ Since $X \subseteq Y$, one can have $X \cap Y=X$. Then, $F R_{A+B}(X \cap Y)=F R_{A+B}(X)$. Besides, it can be found that

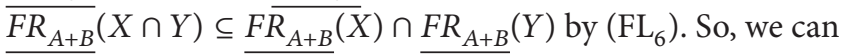

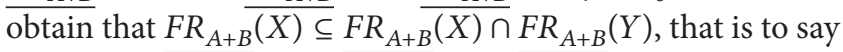

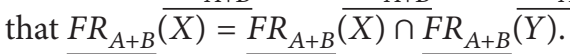

Thus, $F R_{A+B}\left(\overline{X) \subseteq F} R_{A+B}(\overline{Y)}\right.$.

$\left(\mathrm{FU}_{7}\right) \overline{\text { Since }} X \subseteq \overline{Y \text {, one }}$ can have $X \cup Y=Y$. Then, $\overline{F R_{A+B}}(X \cup Y)=\overline{F R_{A+B}}(Y)$. In addition, it can be found that $\overline{F R_{A+B}}(X \cup Y) \supseteq \overline{F R_{A+B}}(X) \cup \overline{F R_{A+B}}(Y)$ by $\left(\mathrm{FU}_{6}\right)$. So, we can obtain that $\overline{F R_{A+B}}(Y) \supseteq \overline{F R_{A+B}}(X) \cup \overline{F R_{A+B}}(Y)$, that is to say that $\overline{F R_{A+B}}(Y)=\overline{F R_{A+B}}(X) \cup \overline{F R_{A+B}}(Y)$.

Thus, $\overline{F R_{A+B}}(X) \subseteq \overline{F R_{A+B}}(Y)$.

$\left(\mathrm{FU}_{8}\right)$ Since $X \subseteq X \cup Y$ and $Y \subseteq X \cup Y$, by $\left(\mathrm{FL}_{7}\right)$ it can be obtained that

$$
\begin{aligned}
& F R_{A+B}(X) \subseteq \underline{F R_{A+B}}(X \cup Y), \\
& F R_{A+B}(Y) \subseteq \underline{F R_{A+B}}(X \cup Y) .
\end{aligned}
$$

So, we have $F R_{A+B}(X) \cup F R_{A+B}(Y) \subseteq F R_{A+B}(X \cup Y)$.

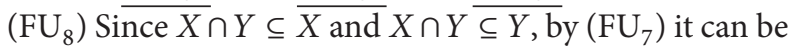
obtained that

$$
\begin{aligned}
& \overline{F R_{A+B}}(X \cap Y) \subseteq \overline{F R_{A+B}}(X), \\
& \overline{F R_{A+B}}(Y \cap Y) \subseteq \overline{F R_{A+B}}(Y) .
\end{aligned}
$$

So, we have $\overline{F R_{A+B}}(X \cap Y) \subseteq \overline{F R_{A+B}}(X) \cap \overline{F R_{A+B}}(X)$.

The proposition was proved.

Here, we employ an example to illustrate the previos concepts and properties with respect to the 1st TGRS.

Example 4. Table 1 depicts an information system containing some information about an emporium investment project. Locus, investment, and population density are the conditional attributes of the systems, whereas Decision is the decision attribute. In the sequel, $L, I, P$, and $D$ will stand for locus, investment, population density and $\{$ Decision $\}$, respectively. The domains are as follows: $V_{L}=\{\mathrm{B}-\mathrm{Bad}, \mathrm{C}-$ Common, G-Good, $\mathrm{G}^{+}$-Better $\}, V_{L}=\{\mathrm{H}-$ High, L-Low $\}, V_{P}=$ $\{\mathrm{B}-$ Big, S-Small, $\mathrm{M}-$ Medium $\}$, and $V_{D}=\{\mathrm{Y}-$ Yes, $\mathrm{N}-$ No\}.

From the table, we can find that

$$
\begin{gathered}
R_{L}=\left\{\left\{u_{1}, u_{7}\right\},\left\{u_{2}, u_{8}\right\},\left\{u_{3}, u_{4}\right\},\left\{u_{5}, u_{6}\right\}\right\}, \\
R_{P}=\left\{\left\{u_{1}, u_{2}\right\},\left\{u_{3}, u_{4}, u_{5}\right\},\left\{u_{6}, u_{7}, u_{8}\right\}\right\}, \\
R_{L \cup P}=\left\{\left\{u_{1}\right\},\left\{u_{2}\right\},\left\{u_{3}, u_{4}\right\},\left\{u_{5}\right\},\left\{u_{6}\right\},\left\{u_{7}\right\},\left\{u_{8}\right\}\right\} .
\end{gathered}
$$

And, if we take $X=\left\{u_{1}, u_{2}, u_{6}, u_{8}\right\}$, then by computing we have

$$
\begin{gathered}
\frac{F R_{L+P}}{}(X)=\left\{u_{1}, u_{2}, u_{8}\right\}, \\
\overline{F R_{L+P}}(X)=\left\{u_{1}, u_{2}, u_{6}, u_{7}, u_{8}\right\} .
\end{gathered}
$$

However, the lower approximation and upper approximation of $X$ based on Pawlak's rough set are

$$
\begin{gathered}
\underline{R_{L}}(X)=\left\{u_{2}, u_{8}\right\}, \\
\overline{R_{L}}(X)=\left\{u_{1}, u_{2}, u_{5}, u_{6}, u_{7}, u_{8}\right\}, \\
\underline{R_{P}}(X)=\left\{u_{1}, u_{2}\right\}, \\
\overline{R_{P}}(X)=\left\{u_{1}, u_{2}, u_{6}, u_{7}, u_{8}\right\}, \\
\frac{R_{L \cup P}}{\overline{R_{L \cup P}}}(X)=\left\{u_{1}, u_{2}, u_{6}, u_{8}\right\},
\end{gathered}
$$

Obviously, one can check the following properties.

$$
\begin{gathered}
\underline{R_{L}}(X) \cup \underline{R_{P}}(X)=\underline{F R_{L+P}}(X), \\
\overline{R_{L}}(X) \cap \overline{R_{P}}(X)=\overline{F R_{L+P}}(X), \\
\underline{F R_{L+P}}(X) \subseteq \underline{R_{L \cup P}}(X) \subseteq X \subseteq \overline{R_{L \cup P}}(X) \subseteq \overline{F R_{L+P}}(X) .
\end{gathered}
$$

3.2. The First Type of Multiple Granulation Rough Set. In this subsection, we will consider the first type of multiple granulation approximations of a target set by using multiple equivalence relations in an information system.

Definition 5. Let $\mathscr{I}=(U, A T, V, f)$ be an information system, $A_{1}, A_{2}, \ldots, A_{s} \subseteq A T$ be attribute subsets $\left(s \leq 2^{|A T|}\right)$, and $R_{A_{1}}, R_{A_{2}}, \ldots, R_{A_{s}}$ be equivalence relations, respectively. The operators $F R_{\sum_{i=1}^{s} A_{i}}$ and $\overline{F R_{\sum_{i=1}^{s} A_{i}}}: \mathscr{P}(U) \rightarrow \mathscr{P}(U)$ are defined as follows. For all $X \in \mathscr{P}(U)$,

$$
\begin{gathered}
\underline{F R_{\sum_{i=1}^{s} A_{i}}}(X)=\left\{u \mid \bigvee_{i=1}^{m}\left([u]_{A_{i}} \subseteq X\right)\right\}, \\
\overline{F R_{\sum_{i=1}^{s} A_{i}}}(X)=\left\{u \mid \bigwedge_{i=1}^{m}\left([u]_{A_{i}} \cap X \neq \emptyset\right)\right\},
\end{gathered}
$$

where " $\vee$ " means "some", and “ $\wedge$ " means "all." We call them the first type of multiple granulation lower and upper approximation operators, and we call $F R_{\sum_{i=1}^{s} A_{i}}(X)$ and $\overline{F R_{\sum_{i=1}^{s} A_{i}}}(X)$ the first type of multiple granulation lower approximation set and upper approximation of $X$, respectively.

Moreover, if $F R_{\sum_{i=1}^{s} A_{i}}(X) \neq \overline{F R_{\sum_{i=1}^{s} A_{i}}}(X)$, we say that $X$ is the first type of rough set with respect to multiple granulation spaces $A_{1}, A_{2}, \ldots, A_{s}$. Otherwise, we say that $X$ is the first type of definable set with respect to these multiple granulation spaces. 
TABLE 1: An information system about emporium investment project.

\begin{tabular}{lcccc}
\hline Project & Locus & Investment & Population density & Decision \\
\hline$u_{1}$ & $\mathrm{C}$ & $\mathrm{L}$ & $\mathrm{B}$ & $\mathrm{N}$ \\
$u_{2}$ & $\mathrm{G}^{+}$ & $\mathrm{H}$ & $\mathrm{B}$ & $\mathrm{Y}$ \\
$u_{3}$ & $\mathrm{~B}$ & $\mathrm{~L}$ & $\mathrm{~S}$ & $\mathrm{~N}$ \\
$u_{4}$ & $\mathrm{~B}$ & $\mathrm{H}$ & $\mathrm{S}$ & $\mathrm{N}$ \\
$u_{5}$ & $\mathrm{G}$ & $\mathrm{L}$ & $\mathrm{S}$ & $\mathrm{N}$ \\
$u_{6}$ & $\mathrm{G}$ & $\mathrm{H}$ & $\mathrm{M}$ & $\mathrm{Y}$ \\
$u_{7}$ & $\mathrm{C}$ & $\mathrm{H}$ & $\mathrm{M}$ & $\mathrm{Y}$ \\
$u_{8}$ & $\mathrm{G}^{+}$ & $\mathrm{H}$ & $\mathrm{M}$ & $\mathrm{Y}$ \\
\hline
\end{tabular}

Similarly, the area of uncertainty or boundary region of this rough set is defined as

$$
\operatorname{Bnd}_{R_{\sum_{i=1}^{s} A_{i}}^{F}}(X)=\overline{F R_{\sum_{i=1}^{s} A_{i}}}(X)-\underline{F R_{\sum_{i=1}^{s} A_{i}}}(X) .
$$

To describe conveniently the ideas in our context, we express the first type of multiple granulation rough set by using the 1st MGRS. Moreover, one can obtain the following properties of the 1st MGRS approximations.

Proposition 6. Let $\mathscr{I}=(U, A T, V, f)$ be an information system, $A_{i} \subseteq A T, i=1,2, \ldots s$, and $X \subseteq U$. Then the following properties hold.

$$
\begin{aligned}
& \left(\mathrm{FL}_{1}\right) \mathrm{FR}_{\sum_{i=1}^{s} A_{i}}(X) \subseteq X \quad \text { (Contraction), } \\
& \left(\mathrm{FU}_{1}\right) \overline{F R_{\sum_{i=1}^{s} A_{i}}}(X) \supseteq X \quad \text { (Extension), } \\
& \left(\mathrm{FL}_{2}\right) \underline{F R_{\sum_{i=1}^{s} A_{i}}}(\sim X)=\sim \overline{F R_{\sum_{i=1}^{s} A_{i}}}(X) \quad \text { (Duality), } \\
& \left(\mathrm{FU}_{2}\right) \overline{F R_{\sum_{i=1}^{s} A_{i}}}(\sim X)=\sim \mathcal{F R _ { \sum _ { i = 1 } ^ { s } A _ { i } }}(X) \quad \text { (Duality), } \\
& \left(\mathrm{FL}_{3}\right) F R_{\sum_{i=1}^{s} A_{i}}(\emptyset)=\emptyset \quad \text { (Normality), } \\
& \left(\mathrm{FU}_{3}\right) \overline{F R_{\sum_{i=1}^{s} A_{i}}}(\emptyset)=\emptyset \quad \text { (Normality), } \\
& \left(\mathrm{FL}_{4}\right) F R_{\sum_{i=1}^{s} A_{i}}(U)=U \quad \text { (Conormality), } \\
& \left(\mathrm{FU}_{4}\right) \overline{F R_{\sum_{i=1}^{s} A_{i}}}(U)=U \quad \text { (Conormality), } \\
& \left(\mathrm{FL}_{5}\right) F R_{\sum_{i=1}^{s} A_{i}}(X)=\underline{F R_{B+A}}(X) \quad \text { (Commutativity), } \\
& \left(\mathrm{FU}_{5}\right) \overline{F R_{\sum_{i=1}^{s} A_{i}}}(X)=\overline{F R_{B+A}}(X) \quad \text { (Commutativity). }
\end{aligned}
$$

Proof. The proof of these items is similar to Proposition 2.

Proposition 7. Let $\mathscr{I}=(U, A T, V, f)$ be an information system, $A_{i} \subseteq A T, i=1,2, \ldots s$ and $X, Y \subseteq U$. Then the following properties hold.

$$
\begin{aligned}
& \left(\mathrm{FL}_{6}\right) F R_{\sum_{i=1}^{s} A_{i}}(X \cap Y) \subseteq F R_{\sum_{i=1}^{s} A_{i}}(X) \cap F R_{\sum_{i=1}^{s} A_{i}}(Y) \quad(L- \\
& \overline{\text { Multiplication), }} \\
& \left(\mathrm{FU}_{6}\right) \overline{F R_{\sum_{i=1}^{s} A_{i}}}(X \cup Y) \supseteq \overline{F R_{\sum_{i=1}^{s} A_{i}}}(X) \cup \overline{F R_{\sum_{i=1}^{s} A_{i}}}(Y) \quad(L-
\end{aligned}
$$

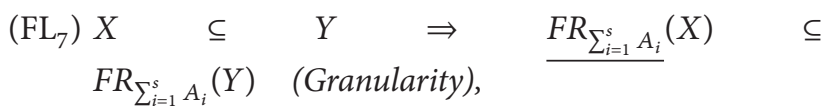

$$
\begin{aligned}
& \left(\mathrm{FU}_{7}\right) X \quad \subseteq \quad Y \quad \Rightarrow \quad F R_{\sum_{i=1}^{s} A_{i}}(X) \quad \subseteq \\
& F R_{\sum_{i=1}^{s} A_{i}}(Y) \text { (Granularity), }
\end{aligned}
$$

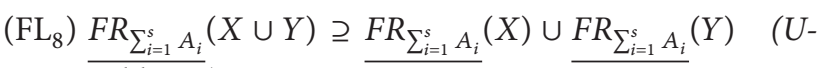

$$
\begin{aligned}
& \text { Addition), } \\
& \left(\mathrm{FU}_{8}\right) \overline{F R_{\sum_{i=1}^{s} A_{i}}}(X \cap Y) \subseteq \overline{F R_{\sum_{i=1}^{s} A_{i}}}(X) \cap \overline{F R_{\sum_{i=1}^{s} A_{i}}}(Y) \\
& \text { Multiplication). }
\end{aligned}
$$

Proof. The proof of these items is similar to Proposition 3.

Next, we will investigate several elementary measures in the 1st MGRS and their properties.

Uncertainty of a set (category) is due to the existence of a borderline region. The bigger the borderline region of a set is, the lower the accuracy of the set is (and vice versa). To more precisely express this idea, we introduce the accuracy measure to the 1st MGRS as follows.

Definition 8. Let $\mathscr{I}=(U, A T, V, f)$ be an information system, $A_{i} \subseteq A T, i=1,2, \ldots s$, and $X \subseteq U$. The 1st rough measure of $X$ by $\sum_{i=1}^{s} A_{i}$ is defined as

$$
\rho_{\sum_{i=1}^{s} A_{i}}^{F}(X)=1-\frac{\left|F R_{\sum_{i=1}^{s} A_{i}}(X)\right|}{\left|\overline{F R_{\sum_{i=1}^{s} A_{i}}}(X)\right|},
$$

where $X \neq \emptyset$.

From the definitions, one can derive the following properties.

Proposition 9. Let $\mathscr{I}=(U, A T, V, f)$ be an information system, $A_{i} \subseteq A T, i=1,2, \ldots s$, and $X \subseteq U$. Then

$$
\rho_{A_{i}}(X) \geq \rho_{\sum_{i=1}^{s} A_{i}}^{F}(X) \geq \rho_{\cup_{i=1}^{s} A_{i}}(X) .
$$

Proof. By Corollary 32, we have

$$
\begin{aligned}
& \overline{R_{A_{i}}}(X) \subseteq \overline{F R_{\sum_{i=1}^{s} A_{i}}}(X), \\
& \overline{R_{A_{i}}}(X) \supseteq \overline{F R_{\sum_{i=1}^{s} A_{i}}}(X) .
\end{aligned}
$$

And by Corollary 40, we have

$$
\begin{aligned}
& \overline{F R_{\sum_{i=1}^{s} A_{i}}}(X) \subseteq \overline{R_{\cup_{i=1}^{s} A_{i}}}(X), \\
& \overline{F R_{\sum_{i=1}^{s} A_{i}}}(X) \supseteq \overline{R_{\cup_{i=1}^{s} A_{i}}}(X) .
\end{aligned}
$$


So, the following hold:

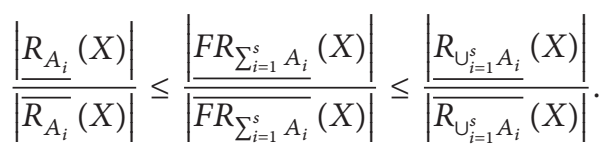

Hence, by the Definition 8 , we have

$$
\rho_{A_{i}}(X) \geq \rho_{\sum_{i=1}^{s} A_{i}}^{F}(X) \geq \rho_{\cup_{i=1}^{s} A_{i}}(X) .
$$

The proof was completed.

Example 10 (continued from Example 4). Computing the 1st rough measures of $X=\left\{u_{1}, u_{2}, u_{6}, u_{8}\right\}$ by using the results in Example 4, it follows that

$$
\begin{aligned}
& \rho_{L}(X)=1-\frac{\left|R_{L}(X)\right|}{\left|\overline{R_{L}}(X)\right|}=\frac{2}{3}, \\
& \rho_{P}(X)=1-\frac{\left|R_{P}(X)\right|}{\left|\overline{R_{P}}(X)\right|}=\frac{3}{5}, \\
& \rho_{L \cup P}(X)=1-\frac{\left|\frac{R_{L \cup P}}{}(X)\right|}{\left|\overline{R_{L \cup P}}(X)\right|}=0, \\
& \rho_{L+P}^{F}(X)=1-\frac{\left|F R_{L+P}(X)\right|}{\left|\overline{F R_{L+P}}(X)\right|}=\frac{2}{5} .
\end{aligned}
$$

Clearly, it follows from the earlier computation that

$$
\begin{aligned}
& \rho_{L}(X) \geq \rho_{L+P}^{F}(X) \geq \rho_{L \cup P}(X), \\
& \rho_{P}(X) \geq \rho_{L+P}^{F}(X) \geq \rho_{L \cup P}(X) .
\end{aligned}
$$

Note that the rough measure of a target concept defined by using multiple granulations is always much better than that defined by using a single granulation, which is suitable for more precisely characterizing a target concept and problem solving according to user requirements.

Definition 11. Let $\mathscr{I}=(U, C \cup\{d\}, V, f)$ be a decision table, $A_{i} \subseteq C, i=1,2, \ldots, s$, and, $\left\{D_{1}, D_{2}, \ldots, D_{k}\right\}$ be all decision classes induced by decision attribute $d$. Approximation quality of $d$ by $\sum_{i=1}^{s} A_{i}$, called the 1st degree of dependence, is defined as

$$
\gamma_{F}\left(\sum_{i=1}^{s} A_{i}, d\right)=\frac{1}{|U|} \sum_{j=1}^{k}\left(\left|F R_{\sum_{i=1}^{s} A_{i}}\left(D_{j}\right)\right|\right) .
$$

This measure can be used to evaluate the deterministic part of the rough set description of $U / d$ by counting those objects which can be reclassified as blocks of $U / d$ with the knowledge given by $\sum_{i=1}^{s} A_{i}$. Moreover, we have the following properties with respect to the above definition.

Proposition 12. Let $\mathscr{I}=(U, C \cup\{d\}, V, f)$ be a decision table, $A_{i} \subseteq C, i=1,2, \ldots, s$, and let $\left\{D_{1}, D_{2}, \ldots, D_{k}\right\}$ be all decision classes induced by decision attribute $d$. Then

$$
\gamma\left(A_{i}, d\right) \leq \gamma_{F}\left(\sum_{i=1}^{s} A_{i}, d\right) \leq \gamma\left(\bigcup_{i=1}^{s} A_{i}, d\right) .
$$

Proof. For every $D_{j}, j=1,2, \ldots, k$, by Corollaries 32 and 40, we have

$$
\underline{R_{A_{i}}}\left(D_{j}\right) \subseteq \underline{F R_{\sum_{i=1}^{s} A_{i}}}\left(D_{j}\right) \subseteq R_{\mathrm{\cup}_{i=1}^{s} A_{i}}\left(D_{j}\right) .
$$

So,

$$
\left|\underline{R_{A_{i}}}\left(D_{j}\right)\right| \leq\left|F R_{\sum_{i=1}^{s} A_{i}}\left(D_{j}\right)\right| \leq\left|\underline{R_{\cup_{i=1}^{s} A_{i}}}\left(D_{j}\right)\right| .
$$

Hence, by Definition 11, we have

$$
\gamma\left(A_{i}, d\right) \leq \gamma_{F}\left(\sum_{i=1}^{s} A_{i}, d\right) \leq \gamma\left(\bigcup_{i=1}^{s} A_{i}, d\right) .
$$

The proof was completed.

Example 13 (continued from Example 4). Computing the degree of dependence of $X=\left\{u_{1}, u_{2}, u_{6}, u_{8}\right\}$.

From Table 1, we can have $U / D=\left\{D_{Y}, D_{N}\right\}$ and

$$
\begin{aligned}
& D_{Y}=\left\{u_{2}, u_{6}, u_{7}, u_{8}\right\}, \\
& D_{N}=\left\{u_{1}, u_{3}, u_{4}, u_{5}\right\} .
\end{aligned}
$$

Moreover, the following can be computed by Table 1 and the results of Example 4,

$$
\begin{aligned}
& \underline{R_{L}}\left(D_{Y}\right)=\left\{u_{2}, u_{8}\right\}, \\
& \underline{R_{P}}\left(D_{Y}\right)=\left\{u_{6}, u_{7}, u_{8}\right\}, \\
& \underline{R_{L \cup P}}\left(D_{Y}\right)=\left\{u_{2}, u_{6}, u_{7}, u_{8}\right\}, \\
& \underline{F R_{L+P}}\left(D_{Y}\right)=\left\{u_{2}, u_{6}, u_{7}, u_{8}\right\}, \\
& \underline{R_{L}}\left(D_{N}\right)=\left\{u_{3}, u_{4}\right\}, \\
& \underline{R_{P}}\left(D_{N}\right)=\left\{u_{3}, u_{4}, u_{5}\right\} \text {, } \\
& \underline{R_{L \cup P}}\left(D_{N}\right)=\left\{u_{1}, u_{3}, u_{4}, u_{5}\right\}, \\
& F_{L+P}\left(D_{N}\right)=\left\{u_{3}, u_{4}, u_{5}\right\} .
\end{aligned}
$$

So, we have

$$
\begin{gathered}
\gamma(L, D)=\frac{1}{|U|}\left(\left|\underline{R_{L}}\left(D_{Y}\right)\right|+\left|\underline{R_{L}}\left(D_{N}\right)\right|\right)=\frac{1}{2}, \\
\gamma(P, D)=\frac{1}{|U|}\left(\underline{\mid R_{P}}\left(D_{Y}\right)|+| \underline{R_{P}}\left(D_{N}\right) \mid\right)=\frac{3}{4}, \\
\gamma(L \cup P, D)=\frac{1}{|U|}\left(\left|\underline{R_{L \cup P}}\left(D_{Y}\right)\right|+\left|\underline{R_{L \cup P}}\left(D_{N}\right)\right|\right)=1, \\
\gamma_{F}(L+P, D)=\frac{1}{|U|}\left(\left|\underline{F R_{L+P}}\left(D_{Y}\right)\right|+\left|\underline{F R_{L+P}}\left(D_{N}\right)\right|\right)=\frac{7}{8} .
\end{gathered}
$$

Hence, it can be found that

$$
\begin{aligned}
& \gamma(L, D) \leq \gamma_{F}(L+P, D) \leq \gamma(L \cup P, D), \\
& \gamma(P, D) \leq \gamma_{F}(L+P, D) \leq \gamma(L \cup P, D) .
\end{aligned}
$$




\section{The Second Type of Multiple Granulation Rough Set}

In this section, we will consider another multiple granulation rough set.

4.1. The Second Type of Two Granulation Rough Set. We first discuss the second type of two granulation approximations of a target set by using two equivalence relations in an information system.

Definition 14. Let $\mathscr{I}=(U, A T, V, f)$ be an information system and $B, A \subseteq A T$. The operators $S R_{A+B}$ and $\overline{S R_{A+B}}$ : $\mathscr{P}(U) \rightarrow \mathscr{P}(U)$ are defined as follows. For all $X \in \mathscr{P}(U)$,

$$
\begin{gathered}
\underline{F R_{A+B}}(X)=\left\{u \mid[u]_{A} \subseteq X,[u]_{B} \subseteq X\right\}, \\
\overline{F R_{A+B}}(X)=\left\{u \mid[u]_{A} \cap X \neq \emptyset, \text { or }[u]_{B} \cap X \neq \emptyset\right\} .
\end{gathered}
$$

We call them the second type of two granulation lower and upper approximation operators, and we call $S R_{A+B}(X)$ and $\overline{S R_{A+B}}(X)$ the second type of two granulation lower approximation set and upper approximation of $X$, respectively.

Moreover, if $S R_{A+B}(X) \neq \overline{S R_{A+B}}(X)$, we say that $X$ is the second type of $\overline{\text { rough }}$ set with respect to two granulation spaces $A$ and $B$. Otherwise, we say that $X$ is the second type of definable set with respect to two granulation spaces $A$ and $B$.

The area of uncertainty or boundary region of this rough set is defined as

$$
\operatorname{Bnd}_{R_{A+B}}^{S}(X)=\overline{S R_{A+B}}(X)-\underline{S R_{A+B}}(X) .
$$

It can be found that the second two granulation rough set will be Pawlak's rough set when two granulation spaces $A$ and $B$ satisfy $A=B$. To describe conveniently in our context, we express the second type of two granulation rough set by using the 2nd TGRS.

By the previos definition, it can be seen that the 2nd TGRS lower and upper approximations are consistent with Pawlak's rough set. Furthermore, one can find that the 2nd TGRS lower and upper approximations are defined through using the equivalence classes induced by multi equivalence relations in an information system, whereas Pawlak's lower and upper approximations are represented via those derived by only one equivalence relation. And the 2nd TGRS lower and upper approximations are dual with the 2nd TGRS lower and upper approximations.

One can understand the second two granulation rough set and show the difference between the 1st TGRS and Pawlak's rough set through Figure 3.

Just from Definition 14, we can obtain the following properties in the 2 nd TGRS in an information system.

Proposition 15. Let $\mathscr{I}=(U, A T, V, f)$ be an information system, $B, A \subseteq A T$ and $X \subseteq U$. Then the following properties hold.

$$
\left(\mathrm{SL}_{1}\right) \mathrm{SR}_{A+B}(X) \subseteq X \quad \text { (Contraction), }
$$
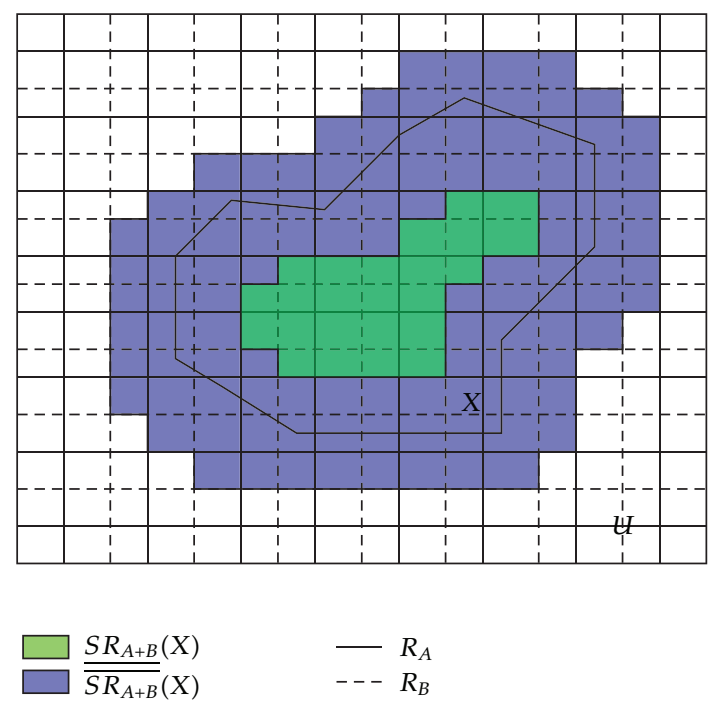

FIGURE 3: The 2nd TGRS in an information system.

$\left(\mathrm{SU}_{1}\right) \overline{\mathrm{SR}} \mathrm{A+B}_{\mathrm{A}}(X) \supseteq X \quad$ (Extension),

$\left(\mathrm{SL}_{2}\right) \underline{S R_{A+B}}(\sim X)=\sim \overline{S R_{A+B}}(X) \quad$ (Duality),

$\left(\mathrm{SU}_{2}\right) \overline{S R_{A+B}}(\sim X)=\sim \underline{S R}_{A+B}(X) \quad$ (Duality),

$\left(\mathrm{SL}_{3}\right) \underline{S R_{A+B}}(\emptyset)=\emptyset \quad$ (Normality),

$\left(\mathrm{SU}_{3}\right) \overline{S R_{A+B}}(\emptyset)=\emptyset \quad$ (Normality),

$\left(\mathrm{SL}_{4}\right) S R_{A+B}(U)=U \quad$ (Conormality),

$\left(\mathrm{SU}_{4}\right) \overline{S R_{A+B}}(U)=U \quad$ (Conormality),

$\left(\mathrm{SL}_{5}\right) \underline{S R_{A+B}}(X)=\underline{S R_{B+A}}(X) \quad$ (Commutativity),

$\left(\mathrm{SU}_{5}\right) \overline{S R_{A+B}}(X)=\overline{S R_{B+A}}(X) \quad$ (Commutativity).

Proof. It is obvious that all terms hold when $A=B$. When $A \neq B$, the proposition can be proved as follows.

$\left(\mathrm{SL}_{1}\right)$ For any $u \in S R_{A+B}(X)$, it can be known that $[u]_{A} \subseteq$ $X$ and $[u]_{B} \subseteq X$ by Definition 14 . However, $u \in[u]_{A}$ and $u \in[u]_{B}$. So we can have $u \in X$. Hence, $S R_{A+B}(X) \subseteq X$.

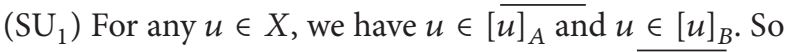
$[u]_{A} \cap X \neq \emptyset$ and $[u]_{B} \cap X \neq \emptyset$, which imply that $u \in \overline{F R_{A+B}}(X)$. Hence, $X \subseteq \overline{S R_{A+B}}(X)$.

$\left(\mathrm{SL}_{2}\right)$ For any $u \in S R_{A+B}(\sim X)$, then

$$
\begin{aligned}
u \in \underline{S_{A+B}}(\sim X) & \Longleftrightarrow[u]_{A} \subseteq \sim X,[u]_{B} \subseteq \sim X, \\
& \Longleftrightarrow[u]_{A} \cap X=\emptyset,[u]_{B} \cap X=\emptyset, \\
& \Longleftrightarrow u \notin \overline{S_{A+B}}(X), \\
& \Longleftrightarrow u \in \sim \overline{S R_{A+B}}(X) .
\end{aligned}
$$

Hence, $S R_{A+B}(\sim \mathrm{X})=\sim \overline{S R_{A+B}}(X)$. 
$\left(\mathrm{SU}_{2}\right)$ By $\left(\mathrm{SL}_{2}\right)$, we have $\underline{S R_{A+B}}(X)=\sim \overline{S R_{A+B}}(\sim X)$. So it can be obtained that $\sim S R_{A+B} \overline{(X)}=\overline{S R_{A+B}}(\sim X)$.

$\left(\mathrm{SL}_{3}\right)$ From $\left(\mathrm{SL}_{1}\right)$, we have $S R_{A+B}(\emptyset) \subseteq \emptyset$. Besides, it is well known that $\emptyset \subseteq S R_{A+B}(\emptyset)$. So, $\overline{S R_{A+B}}(\emptyset)=\emptyset$.

$\left(\mathrm{SU}_{3}\right)$ If $\overline{S R_{A+B}}(\emptyset) \neq \emptyset$, then there must exist a $u \in$ $\overline{S R_{A+B}}(\emptyset)$. So, we can find that $[u]_{A} \cap \emptyset \neq \emptyset$ or $[u]_{B} \cap \emptyset \neq \emptyset$. Obviously, this is a contradiction. Thus, $\overline{S R_{A+B}}(\emptyset)=\emptyset$.

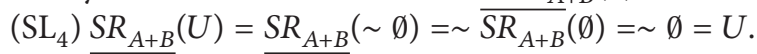
$\left(\mathrm{SU}_{4}\right) \overline{\overline{S R_{A+B}}}(U)=\overline{\overline{S R_{A+B}}}(\sim \emptyset)=\sim S R_{A+B}(\emptyset)=\sim \emptyset=U$.

$\left(\mathrm{SL}_{5}\right)$ and $\left(\mathrm{SU}_{5}\right)$ can be proved directly by Definition 14 .

In order to discover the relationship between the 2nd TGRS approximations of a single set and the 2nd TGRS approximations of two sets described on the universe, the following properties are given.

Proposition 16. Let $\mathscr{I}=(U, A T, V, f)$ be an information system, $B, A \subseteq A T$ and $X, Y \subseteq U$. Then the following properties hold.

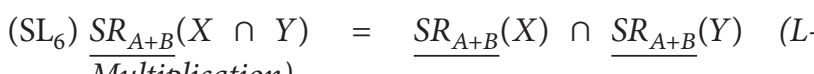

$$
\begin{aligned}
& \text { Multiplication), } \\
& \left(\mathrm{SU}_{6}\right) \overline{S R_{A+B}}(X \cup Y)=\overline{S R_{A+B}}(X) \cup \overline{S R_{A+B}}(Y) \quad \text { (L-Addition), } \\
& \left(\mathrm{SL}_{7}\right) X \subseteq Y \Rightarrow \underline{S R_{A+B}}(X) \subseteq \underline{S R_{A+B}}(Y) \quad \text { (Granularity), } \\
& \left(\mathrm{SU}_{7}\right) X \subseteq Y \Rightarrow S R_{A+B}(X) \subseteq S R_{A+B}(Y) \quad \text { (Granularity), } \\
& \left(\mathrm{SL}_{8}\right) \underline{S R_{A+B}}(X \cup Y) \supseteq \underline{S R_{A+B}}(X) \cup \underline{S R_{A+B}}(Y) \quad \text { (U-Addition), } \\
& \left(\mathrm{SU}_{8}\right) \overline{S R_{A+B}}(X \cap Y) \quad \subseteq \quad \overline{S R_{A+B}}(X) \cap \overline{S R_{A+B}}(Y) \quad(U-
\end{aligned}
$$

Proof. It is obvious that all terms hold when $A=B$ or $X=$ $Y$. When $A \neq B$ and $X \neq Y$, the proposition can be proved as follows.

$\left(\mathrm{SL}_{6}\right)$ For any $u \in S R_{A+B}(X \cap Y)$, by Definition 18 we have

$$
\begin{aligned}
u \in \underline{S R_{A+B}}(X \cap Y) \Longleftrightarrow & {[u]_{A} \subseteq(X \cap Y),[u]_{B} \subseteq(X \cap Y), } \\
\Longleftrightarrow & {[u]_{A} \subseteq X,[u]_{A} \subseteq Y, } \\
& {[u]_{B} \subseteq X,[u]_{B} \subseteq Y } \\
\Longleftrightarrow & {[u]_{A} \subseteq X,[u]_{B} \subseteq X, } \\
& {[u]_{A} \subseteq Y,[u]_{B} \subseteq Y, } \\
\Longleftrightarrow & u \in \underline{S R_{A+B}}(X), u \in \underline{S R_{A+B}}(Y), \\
\Longleftrightarrow & u \in \underline{S R_{A+B}}(X) \cap \underline{S R_{A+B}}(Y)
\end{aligned}
$$

Hence, $\underline{S R_{A+B}}(X \cap Y)=\underline{S R_{A+B}}(X) \cap \underline{S R_{A+B}}(Y)$.
$\left(\mathrm{SU}_{6}\right)$ For any $u \in \overline{S R_{A+B}}(X \cup Y)$, by Definition 18 we have $u \in \overline{S R_{A+B}}(X \cup Y)$

$$
\begin{aligned}
& \Longleftrightarrow[u]_{A} \cap(X \cup Y) \neq \emptyset, \text { or }[u]_{B} \cap(X \cup Y) \neq \emptyset, \\
& \Longleftrightarrow[u]_{A} \cap X \neq \emptyset, \text { or }[u]_{A} \cap Y \neq \emptyset, \\
& \text { or }[u]_{B} \cap X \neq \emptyset \text {, or }[u]_{B} \cap Y \neq \emptyset, \\
& \Longleftrightarrow[u]_{A} \cap X \neq \emptyset \text {, or }[u]_{B} \cap X \neq \emptyset, \\
& \text { or }[u]_{A} \cap Y \neq \emptyset \text {, or }[u]_{B} \cap Y \neq \emptyset, \\
& \Longleftrightarrow u \in \overline{S R_{A+B}}(X) \text {, or } u \in \overline{S R_{A+B}}(Y), \\
& \Longleftrightarrow u \in \underline{S R_{A+B}}(X) \cup \underline{S R_{A+B}}(Y) .
\end{aligned}
$$

Hence, $\overline{S R_{A+B}}(X \cup Y)=\overline{S R_{A+B}}(X) \cup \overline{S R_{A+B}}(Y)$.

$\left(\mathrm{SL}_{7}\right)$ Since $X \subseteq Y$, one can have $X \cap Y=X$. Then, $S R_{A+B}(X \cap Y)=S R_{A+B}(X)$. Besides, it can be found that $\overline{S R_{A+B}}(X \cap Y)=S \overline{R_{A+B}(X)} \cap \underline{S R_{A+B}}(Y)$ by $\left(\mathrm{SL}_{6}\right)$. So, we can $\overline{\text { obtain }}$ that $S R_{A+B} \overline{(X)=} S R_{A+B} \overline{(X) \cap} S R_{A+B}(Y)$, that is to say

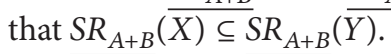

$\left(\overline{\left.\mathrm{SU}_{7}\right)}\right.$ Since $\overline{X \subseteq Y}$, one can have $X \cup Y=Y$. Then, $\overline{S R_{A+B}}(X \cup Y)=\overline{S R_{A+B}}(Y)$. Besides, it can be found that $\overline{S R_{A+B}}(X \cup Y)=\overline{S R_{A+B}}(X) \cup \overline{S R_{A+B}}(Y)$ by $\left(\mathrm{SU}_{6}\right)$. So, we can obtain that $\overline{S R_{A+B}}(Y)=\overline{S R_{A+B}}(X) \cup \overline{S R_{A+B}}(Y)$, that is to say that $\overline{S R_{A+B}}(X) \subseteq \overline{S R_{A+B}}(Y)$.

$\left(\mathrm{SU}_{8}\right)$ Since $X \subseteq X \cup Y$ and $Y \subseteq X \cup Y$, by $\left(\mathrm{SL}_{7}\right)$ it can be obtained that

$$
\begin{aligned}
& S R_{A+B}(X) \subseteq \underline{S R_{A+B}}(X \cup Y), \\
& S R_{A+B}(Y) \subseteq \underline{S R_{A+B}}(X \cup Y) .
\end{aligned}
$$

So, we have $S R_{A+B}(X) \cup S R_{A+B}(Y) \subseteq S R_{A+B}(X \cup Y)$.

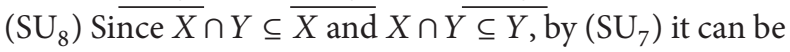
obtained that

$$
\begin{aligned}
& \overline{S R_{A+B}}(X \cap Y) \subseteq \overline{S R_{A+B}}(X), \\
& \overline{S R_{A+B}}(Y \cap Y) \subseteq \overline{S R_{A+B}}(Y) .
\end{aligned}
$$

So, we have $\overline{S R_{A+B}}(X \cap Y) \subseteq \overline{S R_{A+B}}(X) \cap \overline{S R_{A+B}}(X)$.

The proposition was proved.

Example 17 (continued from Example 4). In Example 4, we have known that

$$
\begin{gathered}
R_{L}=\left\{\left\{u_{1}, u_{7}\right\},\left\{u_{2}, u_{8}\right\},\left\{u_{3}, u_{4}\right\},\left\{u_{5}, u_{6}\right\}\right\}, \\
R_{P}=\left\{\left\{u_{1}, u_{2}\right\},\left\{u_{3}, u_{4}, u_{5}\right\},\left\{u_{6}, u_{7}, u_{8}\right\}\right\}, \\
R_{L \cup P}=\left\{\left\{u_{1}\right\},\left\{u_{2}\right\},\left\{u_{3}, u_{4}\right\},\left\{u_{5}\right\},\left\{u_{6}\right\},\left\{u_{7}\right\},\left\{u_{8}\right\}\right\} .
\end{gathered}
$$

And, if we take $X=\left\{u_{1}, u_{2}, u_{6}, u_{8}\right\}$ again, then by computing we have

$$
\begin{aligned}
& \underline{S R_{L+P}}(X)=\left\{u_{2}\right\}, \\
& \overline{S R_{L+P}}(X)=\left\{u_{1}, u_{2}, u_{5}, u_{6}, u_{7}, u_{8}\right\} .
\end{aligned}
$$


However, the lower approximation and upper approximation of $X$ based on Pawlak's rough set were obtained in Example 4 as follows:

$$
\begin{gathered}
\underline{R_{L}}(X)=\left\{u_{2}, u_{8}\right\}, \\
\overline{R_{L}}(X)=\left\{u_{1}, u_{2}, u_{5}, u_{6}, u_{7}, u_{8}\right\}, \\
\underline{R_{P}}(X)=\left\{u_{1}, u_{2}\right\}, \\
\overline{R_{P}}(X)=\left\{u_{1}, u_{2}, u_{6}, u_{7}, u_{8}\right\}, \\
\frac{R_{L \cup P}}{\overline{R_{L \cup P}}}(X)=\left\{u_{1}, u_{2}, u_{6}, u_{8}\right\}, \\
\text { (X) }
\end{gathered}
$$

So, one can check the following properties.

$$
\begin{aligned}
& \underline{R_{L}}(X) \cap \underline{R_{P}}(X)=\underline{F R_{L+P}}(X), \\
& \overline{R_{L}}(X) \cup \overline{R_{P}}(X)=\overline{S R_{L+P}}(X) \text {, } \\
& \underline{F R_{L+P}}(X) \subseteq \underline{R_{L \cup P}}(X) \subseteq X \subseteq \overline{R_{L \cup P}}(X) \subseteq \overline{F R_{L+P}}(X) .
\end{aligned}
$$

4.2. The Second Type of Multiple Granulation Rough Set. In this subsection, we will consider the second type of multiple granulation approximations of a target set by using multiple equivalence relations in an information system.

Definition 18. Let $\mathscr{I}=(U, A T, V, f)$ be an information system, $A_{1}, A_{2}, \ldots, A_{s} \subseteq A T$ attribute subsets $\left(s \leq 2^{|A T|}\right)$, and $R_{A_{1}}, R_{A_{2}}, \ldots, R_{A_{s}}$ equivalence relations, respectively. The operators $S R_{\sum_{i=1}^{s} A_{i}}$ and $\overline{S R_{\sum_{i=1}^{s} A_{i}}}: \mathscr{P}(U) \rightarrow \mathscr{P}(U)$ are defined as follows. For all $X \in \mathscr{P}(U)$,

$$
\begin{gathered}
\underline{S R_{\sum_{i=1}^{s} A_{i}}}(X)=\left\{u \mid \bigwedge_{i=1}^{m}\left([u]_{A_{i}} \subseteq X\right)\right\}, \\
\overline{S R_{\sum_{i=1}^{s} A_{i}}}(X)=\left\{u \mid \bigvee_{i=1}^{m}\left([u]_{A_{i}} \cap X \neq \emptyset\right)\right\},
\end{gathered}
$$

where " $\vee$ " means "some" and " $\wedge$ " means "all." We call them the second type of multiple granulation lower and upper approximation operators, and we call $S R_{\sum_{i=1}^{s} A_{i}}(X)$ and $\overline{S R_{\sum_{i=1}^{s} A_{i}}}(X)$ the second type of multiple $\overline{\text { granulation }}$ lower approximation set and upper approximation of $X$, respectively.

Moreover, if $S R_{\sum_{i=1}^{s} A_{i}}(X) \neq \overline{S R_{\sum_{i=1}^{s} A_{i}}}(X)$, we say that $X$ is the second type of rough set with respect to multiple granulation spaces $A_{1}, A_{2}, \ldots, A_{s}$. Otherwise, we say that $X$ is the second type of definable set with respect to these multiple granulation spaces.

Similarly, the area of uncertainty or boundary region of this rough set is defined as

$$
\operatorname{Bnd}_{R_{\sum_{i=1}^{s} A_{i}}^{S}}(X)=\overline{S R_{\sum_{i=1}^{s} A_{i}}}(X)-\underline{S R_{\sum_{i=1}^{s} A_{i}}}(X) .
$$

To describe conveniently in our context, we express the second type of multiple granulation rough set by using the 2nd MGRS. Moreover, one can obtain the following properties of the 2 nd MGRS approximations.

Proposition 19. Let $\mathscr{I}=(U, A T, V, f)$ be an information system, $A_{i} \subseteq A T, i=1,2, \ldots s$, and $X \subseteq U$. Then the following properties hold.
$\left(\mathrm{SL}_{1}\right) \quad S R_{\sum_{i=1}^{s} A_{i}}(X) \subseteq X$
(Contraction),
$\left(\mathrm{SU}_{1}\right) \overline{\overline{\operatorname{SR}_{\sum_{i=1}^{s} A_{i}}}}(X) \supseteq X$
(Extension),
$\left(\mathrm{SL}_{2}\right) \quad S R_{\sum_{i=1}^{s} A_{i}}(\sim X)=\sim \overline{S R_{\sum_{i=1}^{s} A_{i}}}(X)$ (Duality),
$\left(\mathrm{SU}_{2}\right) \overline{\overline{S R_{\sum_{i=1}^{s} A_{i}}}}(\sim X)=\sim S R_{\sum_{i=1}^{s} A_{i}}(X)$ (Duality),
$\left(\mathrm{SL}_{3}\right) \quad S R_{\sum_{i=1}^{s} A_{i}}(\emptyset)=\emptyset$
(Normality),
$\left(\mathrm{SU}_{3}\right) \overline{\overline{S R_{\sum_{i=1}^{s} A_{i}}}}(\emptyset)=\emptyset$
(Normality),
$\left(\mathrm{SL}_{4}\right) \quad S R_{\sum_{i=1}^{s} A_{i}}(U)=U$
(Conormality),
$\left(\mathrm{SU}_{4}\right) \overline{\overline{S R_{\sum_{i=1}^{s} A_{i}}}}(U)=U$
(Conormality)
$\left(\mathrm{SL}_{5}\right) \quad S R_{\sum_{i=1}^{s} A_{i}}(X)=S R_{B+A}(X)$
(Commutativity),
$\left(\mathrm{SU}_{5}\right) \overline{\overline{S R_{\sum_{i=1}^{s} A_{i}}}}(X)=\overline{\overline{S R_{B+A}}}(X)$
(Commutativity).

Proof. The proof of these items is similar to Proposition 15.

Proposition 20. Let $\mathscr{I}=(U, A T, V, f)$ be an information system, $A_{i} \subseteq A T, i=1,2, \ldots s$, and $X, Y \subseteq U$. Then the following properties hold.

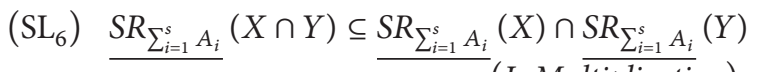

$$
\begin{aligned}
& \left(\mathrm{SU}_{6}\right) \overline{S R_{\sum_{i=1}^{s} A_{i}}}(X \cup Y) \supseteq \overline{S R_{\sum_{i=1}^{s} A_{i}}}(X) \cup \overline{S R_{\sum_{i=1}^{s} A_{i}}}(Y) \\
& \text { (L-Addition), } \\
& \left(\mathrm{SL}_{7}\right) \quad X \subseteq Y \Longrightarrow \underline{S R_{\sum_{i=1}^{s} A_{i}}}(X) \subseteq S R_{\sum_{i=1}^{s} A_{i}}(Y) \\
& \left(\mathrm{SU}_{7}\right) X \subseteq Y \Longrightarrow \underline{S R_{\sum_{i=1}^{s} A_{i}}}(X) \subseteq S R_{\sum_{i=1}^{s} A_{i}}(Y)
\end{aligned}
$$

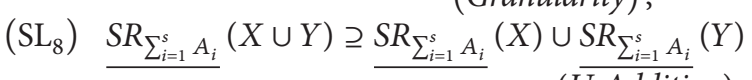

$$
\begin{aligned}
& \left(\mathrm{SU}_{8}\right) \overline{S R_{\sum_{i=1}^{s} A_{i}}}(X \cap Y) \subseteq \overline{S R_{\sum_{i=1}^{s} A_{i}}}(X) \cap \overline{S R_{\sum_{i=1}^{s} A_{i}}}(Y) \\
& \text { (U-Multiplication). }
\end{aligned}
$$

Proof. The proof of these items is similar to Proposition 16.

Next, we will investigate several elementary measures in the 2nd MGRS and their properties.

Similarly, we introduce the accuracy measure to the 2nd MGRS as follows.

Definition 21. Let $\mathscr{I}=(U, A T, V, f)$ be an information system, $A_{i} \subseteq A T, i=1,2, \ldots s$ and $X \subseteq U$. The 2 nd rough measure of $X$ by $\sum_{i=1}^{s} A_{i}$ is defined as

$$
\rho_{\sum_{i=1}^{s} A_{i}}^{S}(X)=1-\frac{\left|S R_{\sum_{i=1}^{s} A_{i}}(X)\right|}{\mid \overline{S R_{\sum_{i=1}^{s} A_{i}}(X) \mid}},
$$

where $X \neq \emptyset$.

From the definitions, one can derive the following properties. 
Proposition 22. Let $\mathscr{I}=(U, A T, V, f)$ be an information system, $A_{i} \subseteq A T, i=1,2, \ldots s$, and $X \subseteq U$. Then

$$
\rho_{\sum_{i=1}^{s} A_{i}}^{S}(X) \geq \rho_{A_{i}}(X) \geq \rho_{\cup_{i=1}^{s} A_{i}}(X) .
$$

Proof. By Corollary 34, we have

$$
\begin{aligned}
& S R_{\sum_{i=1}^{s} A_{i}}(X) \subseteq \underline{R_{A_{i}}}(X), \\
& \overline{S R_{\sum_{i=1}^{s} A_{i}}}(X) \supseteq \overline{R_{A_{i}}}(X) .
\end{aligned}
$$

And, we have known that

$$
\begin{aligned}
& \overline{R_{A_{i}}}(X) \subseteq \underline{R_{\cup_{i=1}^{s} A_{i}}}(X), \\
& \overline{R_{A_{i}}}(X) \supseteq \overline{R_{\cup_{i=1}^{s} A_{i}}}(X) .
\end{aligned}
$$

So, the following holds:

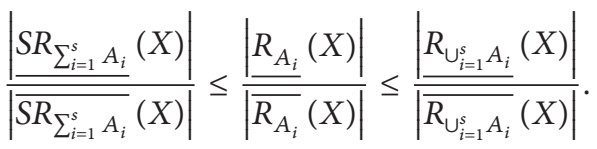

Hence, by the Definition 21, we have

$$
\rho_{\sum_{i=1}^{s} A_{i}}^{S}(X) \geq \rho_{A_{i}}(X) \geq \rho_{\cup_{i=1}^{s} A_{i}}(X) .
$$

The proof was completed.

Example 23 (continued from Examples 4 and 17). Computing the 2 nd rough measures of $X=\left\{u_{1}, u_{2}, u_{6}, u_{8}\right\}$ in the system given in Example 4. By Example 17, it follows that

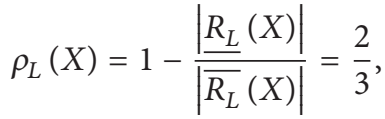

$$
\begin{aligned}
& \rho_{P}(X)=1-\frac{\left|\frac{R_{P}}{}(X)\right|}{\left|\overline{R_{P}}(X)\right|}=\frac{3}{5}, \\
& \rho_{L \cup P}(X)=1-\frac{\left|\frac{R_{L \cup P}}{\mid \overline{R_{L \cup P}}}(X)\right|}{\mid \bar{x}) \mid}=0, \\
& \rho_{L+P}^{S}(X)=1-\frac{\left|\underline{S R_{L+P}}(X)\right|}{\left|\overline{S R_{L+P}}(X)\right|}=\frac{5}{6} \text {. }
\end{aligned}
$$

Clearly, it follows from the earlier computation that

$$
\begin{gathered}
\rho_{L+P}^{S}(X) \geq \rho_{L}(X) \geq \rho_{L \cup P}(X), \\
\rho_{L+P}^{S}(X) \geq \rho_{P}(X) \geq \rho_{L \cup P}(X) .
\end{gathered}
$$

Similar to the 1st MGRS, in the following we will discuss the 2 nd degree of dependence.

Definition 24. Let $\mathscr{I}=(U, C \cup\{d\}, V, f)$ be a decision table, $A_{i} \subseteq C, i=1,2, \ldots, s$, and $\left\{D_{1}, D_{2}, \ldots, D_{k}\right\}$ all decision classes induced by decision attribute $d$. Approximation quality of $d$ by $\sum_{i=1}^{s} A_{i}$, called the 2 nd degree of dependence, is defined as

$$
\gamma_{S}\left(\sum_{i=1}^{s} A_{i}, d\right)=\frac{1}{|U|} \sum_{j=1}^{k}\left(\left|S R_{\sum_{i=1}^{s} A_{i}}\left(D_{j}\right)\right|\right) .
$$

Moreover, we have the following properties with resect to the above definition.

Proposition 25. Let $\mathscr{I}=(U, C \cup\{d\}, V, f)$ be a decision table, $A_{i} \subseteq C, i=1,2, \ldots, s$, and $\left\{D_{1}, D_{2}, \ldots, D_{k}\right\}$ all decision classes induced by decision attribute $d$. Then

$$
\gamma_{S}\left(\sum_{i=1}^{s} A_{i}, d\right) \leq \gamma\left(A_{i}, d\right) \leq \gamma\left(\bigcup_{i=1}^{s} A_{i}, d\right) .
$$

Proof. For every $D_{j}, j=1,2, \ldots, k$, by Corollaries 32 and 40, we have

$$
\underline{S R_{\sum_{i=1}^{s} A_{i}}}\left(D_{j}\right) \subseteq \underline{R_{A_{i}}}\left(D_{j}\right) \subseteq \underline{R_{\cup_{i=1}^{s} A_{i}}}\left(D_{j}\right) .
$$

So,

$$
\left|\underline{S R_{\sum_{i=1}^{s} A_{i}}}\left(D_{j}\right)\right| \leq\left|\underline{R_{A_{i}}}\left(D_{j}\right)\right| \leq\left|\underline{R_{\cup_{i=1}^{s} A_{i}}}\left(D_{j}\right)\right| .
$$

Hence, by the Definition 24, we have

$$
\gamma_{S}\left(\sum_{i=1}^{s} A_{i}, d\right) \leq \gamma\left(A_{i}, d\right) \leq \gamma\left(\bigcup_{i=1}^{s} A_{i}, d\right) .
$$

The proof was completed.

Example 26 (continued from Examples 4 and 17). Computing the 2 nd degree of dependence of $X=\left\{u_{1}, u_{2}, u_{6}, u_{8}\right\}$.

In Example 10, we have known that $U / D=\left\{D_{Y}, D_{N}\right\}$ and

$$
\begin{gathered}
\gamma(L, D)=\frac{1}{2}, \\
\gamma(P, D)=\frac{3}{4}, \\
\gamma(L \cup P, D)=1 .
\end{gathered}
$$

Moreover, the following can be computed by Table 1 and the results of Example 17,

$$
\begin{gathered}
\underline{S R_{L+P}}\left(D_{Y}\right)=\left\{u_{8}\right\}, \\
\underline{S R_{L+P}}\left(D_{N}\right)=\left\{u_{3}, u_{4}\right\} .
\end{gathered}
$$

So, we have

$$
\gamma_{S}(L+P, D)=\frac{1}{|U|}\left(\left|\underline{S R_{L+P}}\left(D_{Y}\right)\right|+\left|\underline{S R_{L+P}}\left(D_{N}\right)\right|\right)=\frac{3}{8} .
$$


Hence, it can be found that

$$
\begin{aligned}
& \gamma_{S}(L+P, D) \leq \gamma(L, D) \leq \gamma(L \cup P, D), \\
& \gamma_{S}(L+P, D) \leq \gamma(P, D) \leq \gamma(L \cup P, D) .
\end{aligned}
$$

\section{Difference and Relationship among Pawlak's Rough Set, and the 1st MGRS, the 2nd MGRS}

From the previos sections, we have known the concepts and properties of the 1st MGRS and the 2nd MGRS. We will investigate the difference and relationship among Pawlak's rough set, the 1st MGRS, and the 2nd MGRS in this section.

Proposition 27. Let $\mathscr{I}=(U, A T, V, f)$ be an information system, $B, A \subseteq A T$, and $X \subseteq U$. Then, the following properties hold.

(1) $F R_{A+B}(X) \subseteq \underline{R_{A \cup B}}(X)$,

(2) $\overline{F R_{A+B}}(X) \supseteq \overline{R_{A \cup B}}(X)$.

Proof. (1) For any $u \in F R_{A+B}(X)$, it can be known that $[u]_{A} \subseteq$ $X$ or $[u]_{B} \subseteq X$ by Definition 1 . On the other hand, since $A \subseteq$ $A \cup B$ and $B \subseteq A \cup B$, we have $[u]_{A \cup B} \subseteq[u]_{A}$ and $[u]_{A \cup B} \subseteq$ $[u]_{B}$. So we can obtain that $[u]_{A \cup B} \subseteq X$. That is to say that $u \in \underline{R_{A \cup B}}(X)$. Hence, $F R_{A+B}(X) \subseteq \underline{R_{A \cup B}}(X)$.

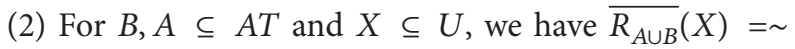
$R_{A \cup B}(\sim X)$. Then, it can be got that $F R_{A+B}(\sim X) \subseteq R_{A \cup B}(\sim X)$ by the conclusion of (1). So, one can obtain that $\overline{\sim F R}_{A+B}(\sim$ $X) \supseteq \sim R_{A \cup B}(\sim X)$. Hence, $\overline{F R_{A+B}}(X) \supseteq \overline{R_{A \cup B}}(X)$.

The proof was completed.

Corollary 28. Let $\mathscr{I}=(U, A T, V, f)$ be an information system, $A_{i} \subseteq A T, i=1,2, \ldots, s$, and $X \subseteq U$. Then, the following properties hold.

(1) $F R_{\sum_{i}^{s} A_{i}}(X) \subseteq \underline{R_{\cup_{i}^{s} A_{i}}}(X)$,

(2) $\overline{\overline{F R_{\sum_{i}^{s} A_{i}}}}(X) \supseteq \overline{R_{\cup_{i}^{s} A_{i}}}(X)$.

Proposition 29. Let $\mathscr{I}=(U, A T, V, f)$ be an information system, $B, A \subseteq A T$, and $X \subseteq U$. Then, the following properties hold.

(1) $\underline{S R_{A+B}}(X) \subseteq \underline{R_{A \cup B}}(X)$,

(2) $\overline{S R_{A+B}}(X) \supseteq \overline{R_{A \cup B}}(X)$.

Proof. (1) For any $u \in S R_{A+B}(X)$, it can be known that $[u]_{A} \subseteq$ $X$ and $[u]_{B} \subseteq X$ by Definition 14. On the other hand, since $A \subseteq A \cup B$ and $B \subseteq A \cup B$, we have $[u]_{A \cup B} \subseteq[u]_{A}$ and $[u]_{A \cup B} \subseteq[u]_{B}$. So we can obtain that $[u]_{A \cup B} \subseteq X$. That is to say that $u \in R_{A \cup B}(X)$. Hence, $S R_{A+B}(X) \subseteq R_{A \cup B}(X)$.

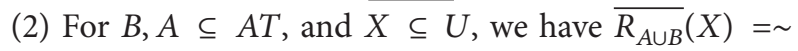
$R_{A \cup B}(\sim X)$. Then, it can be got that $S R_{A+B}(\sim X) \subseteq$ $\overline{R_{A \cup B}}(\sim X)$ by the conclusion of (1). So, one can obtain that ${\overline{\sim S R_{A+B}}}(\sim X) \supseteq \sim R_{A \cup B}(\sim X)$. Hence, $\overline{S R_{A+B}}(X) \supseteq \overline{R_{A \cup B}}(X)$.

The proof was completed.
Corollary 30. Let $\mathscr{I}=(U, A T, V, f)$ be an information system, $A_{i} \subseteq A T, i=1,2, \ldots, s$, and $X \subseteq U$. Then, the following properties hold.
(1) $S R_{\sum_{i}^{s} A_{i}}(X) \subseteq \underline{R_{\cup_{i}^{s} A_{i}}}(X)$,
(2) $\overline{S R_{\sum_{i}^{s} A_{i}}}(X) \supseteq \overline{R_{\cup_{i}^{s} A_{i}}}(X)$.

Proposition 31. Let $\mathscr{I}=(U, A T, V, f)$ be an information system, $B, A \subseteq A T$, and $X \subseteq U$. Then, the following properties hold.

(1) $\underset{F R_{A+B}}{ }(X)=\underline{R_{A}}(X) \cup \underline{R_{B}}(X)$,

(2) $\overline{F R_{A+B}}(X)=\overline{R_{A}}(X) \cap \overline{R_{B}}(X)$.

Proof. (1) For any $u \in F R_{A+B}(X)$, we have

$$
\begin{aligned}
u \in \underline{F R_{A+B}}(X) & \Longleftrightarrow[u]_{A} \subseteq X, \text { or }[u]_{B} \subseteq X, \\
& \Longleftrightarrow u \in \underline{R_{A}}(X), \text { or } u \in \underline{R_{B}}(X), \\
& \Longleftrightarrow u \in \underline{R_{A}}(X) \cup \underline{R_{B}}(X) .
\end{aligned}
$$

Hence, $F R_{A+B}(X)=R_{A}(X) \cup \underline{R_{B}}(X)$.

(2) $\overline{\text { For any }} u \in \overline{F R_{A+B}}(X)$, we have

$$
\begin{aligned}
u \in \overline{F R_{A+B}}(X) & \Longleftrightarrow[u]_{A} \cap X \neq \emptyset,[u]_{B} \cap X \neq \emptyset \\
& \Longleftrightarrow u \in \overline{R_{A}}(X), u \in \overline{R_{B}}(X) \\
& \Longleftrightarrow u \in \overline{R_{A}}(X) \cap \overline{R_{B}}(X) .
\end{aligned}
$$

Hence, $\overline{F R_{A+B}}(X)=\overline{R_{A}}(X) \cap \overline{R_{B}}(X)$.

The proof was completed.

Corollary 32. Let $\mathscr{I}=(U, A T, V, f)$ be an information system, $A_{i} \subseteq A T, i=1,2, \ldots, s$ and $X \subseteq U$. Then, the following properties hold.

(1) $F R_{\sum_{i}^{s} A_{i}}(X)=\bigcup_{i=1}^{s} \underline{R_{A_{i}}}(X)$,

(2) $\overline{F R_{\sum_{i}^{s} A_{i}}}(X)=\bigcap_{i=1}^{s} \overline{R_{A_{i}}}(X)$.

Proposition 33. Let $\mathscr{I}=(U, A T, V, f)$ be an information system, $B, A \subseteq A T$, and $X \subseteq U$. Then, the following properties hold.

(1) $\underline{S R_{A+B}}(X)=\underline{R_{A}}(X) \cap \underline{R_{B}}(X)$,

(2) $\overline{S R_{A+B}}(X)=\overline{R_{A}}(X) \cup \overline{R_{B}}(X)$.

Proof. (1) For any $u \in S R_{A+B}(X)$, we have

$$
\begin{aligned}
u \in \underline{S R_{A+B}}(X) & \Longleftrightarrow[u]_{A} \subseteq X,[u]_{B} \subseteq X, \\
& \Longleftrightarrow u \in \underline{R_{A}}(X), u \in \underline{R_{B}}(X), \\
& \Longleftrightarrow u \in \underline{R_{A}}(X) \cap \underline{R_{B}}(X) .
\end{aligned}
$$

Hence, $\underline{S R_{A+B}}(X)=\underline{R_{A}}(X) \cap \underline{R_{B}}(X)$. 
(2) For any $u \in \overline{S R_{A+B}}(X)$, we have

$$
\begin{aligned}
u \in \overline{S_{A+B}}(X) & \Longleftrightarrow[u]_{A} \cap X \neq \emptyset \text {, or }[u]_{B} \cap X \neq \emptyset, \\
& \Longleftrightarrow u \in \overline{R_{A}}(X), \text { or } u \in \overline{R_{B}}(X), \\
& \Longleftrightarrow u \in \overline{R_{A}}(X) \cup \overline{R_{B}}(X) .
\end{aligned}
$$

Hence, $\overline{S R_{A+B}}(X)=\overline{R_{A}}(X) \cup \overline{R_{B}}(X)$.

The proof was completed.

Corollary 34. Let $\mathscr{I}=(U, A T, V, f)$ be an information system, $A_{i} \subseteq A T, i=1,2, \ldots, s$, and $X \subseteq U$. Then, the following properties hold.

(1) $S R_{\sum_{i}^{s} A_{i}}(X)=\bigcap_{i=1}^{s} \underline{R_{A_{i}}}(X)$,

(2) $\overline{S R_{\sum_{i}^{s} A_{i}}}(X)=\bigcup_{i=1}^{s} \overline{R_{A_{i}}}(X)$.

Proposition 35. Let $\mathscr{I}=(U, A T, V, f)$ be an information system, $B, A \subseteq A T$, and $X, Y \subseteq U$. Then, the following properties hold.

(1) $\underline{F R_{A+B}}(X \cap Y)=\left(\underline{R_{A}}(X) \cap \underline{R_{A}}(Y)\right) \cup\left(\underline{R_{B}}(X) \cap \underline{R_{B}}(Y)\right)$,

(2) $\overline{F R_{A+B}}(X \cup Y)=\left(\overline{R_{A}}(X) \cup \overline{R_{A}}(X)\right) \cap\left(\overline{R_{B}}(X) \cup \overline{R_{B}}(Y)\right)$.

Proof. It can be obtained easily by Proposition 31 .

Corollary 36. Let $\mathscr{I}=(U, A T, V, f)$ be an information system, $A_{i} \subseteq A T, i=1,2, \ldots, s$, and $X, Y \subseteq U$. Then, the following properties hold.

(1) $\underline{F R_{\sum_{i}^{s} A_{i}}}(X \cap Y)=\bigcup_{i=1}^{s}\left(\underline{R_{A_{i}}}(X) \cap \underline{R_{A_{i}}}(Y)\right)$,

(2) $\overline{F R_{\sum_{i}^{s} A_{i}}}(X \cup Y)=\bigcap_{i=1}^{s}\left(\overline{R_{A_{i}}}(X) \cup \overline{R_{A_{i}}}(Y)\right)$.

Proposition 37. Let $\mathscr{I}=(U, A T, V, f)$ be an information system, $B, A \subseteq A T$, and $X, Y \subseteq U$. Then, the following properties hold.

(1) $\underline{S R_{A+B}}(X \cap Y)=\left(\underline{R_{A}}(X) \cap \underline{R_{A}}(Y)\right) \cap\left(\underline{R_{B}}(X) \cap \underline{R_{B}}(Y)\right)$,

(2) $\overline{S R_{A+B}}(X \cup Y)=\left(\overline{R_{A}}(X) \cup \overline{R_{A}}(X)\right) \cup\left(\overline{R_{B}}(X) \cup \overline{R_{B}}(Y)\right)$.

Proof. It can be obtained directly by Proposition 33 .

Corollary 38. Let $\mathscr{I}=(U, A T, V, f)$ be an information system, $A_{i} \subseteq A T, i=1,2, \ldots, s$, and $X, Y \subseteq U$. Then, the following properties hold.

(1) $\operatorname{SR}_{\sum_{i}^{s} A_{i}}(X \cap Y)=\bigcap_{i=1}^{s}\left(\underline{R_{A_{i}}}(X) \cap \underline{R_{A_{i}}}(Y)\right)$,

(2) $\overline{S R_{\sum_{i}^{s} A_{i}}}(X \cup Y)=\bigcup_{i=1}^{s}\left(\overline{R_{A_{i}}}(X) \cup \overline{R_{A_{i}}}(Y)\right)$.

Proposition 39. Let $\mathscr{I}=(U, A T, V, f)$ be an information system, $B, A \subseteq A T$, and $X \subseteq U$. Then, the following properties hold.

(1) $\underline{S R_{A+B}}(X) \subseteq \underline{F R_{A+B}}(X) \subseteq \underline{R_{A \cup B}}(X)$,

(2) $\overline{S R_{A+B}}(X) \supseteq \overline{F R_{A+B}}(X) \supseteq R_{A \cup B}(X)$.
Proof. It can be obtained easily by Definitions 1 and 14 and Propositions 27 and 29.

Corollary 40. Let $\mathscr{I}=(U, A T, V, f)$ be an information system, $A_{i} \subseteq A T, i=1,2, \ldots, s$, and $X \subseteq U$. Then, the following properties hold.
(1) $\underline{S R}_{\sum_{i=1}^{s} A_{i}}(X) \subseteq \underline{F R_{\sum_{i=1}^{s} A_{i}}}(X) \subseteq \underline{R_{\cup_{i=1}^{s} A_{i}}}(X)$,
(2) $\overline{S R_{\sum_{i=1}^{s} A_{i}}}(X) \supseteq \overline{F R_{\sum_{i=1}^{s} A_{i}}}(X) \supseteq{\underline{\mathrm{U}_{i=1}^{s} A_{i}}}(X)$.

Proposition 41. Let $\mathscr{I}=(U, A T, V, f)$ be an information system, $B, A \subseteq A T$, and $X \subseteq U$. Then, the following properties hold.

(1) $S R_{A+B}(X) \subseteq \underline{R_{A}}(X)\left(\right.$ or $\left.\underline{R_{B}}(X)\right) \subseteq F R_{A+B}(X)$,

(2) $\overline{S R_{A+B}}(X) \supseteq R_{A}(X)\left(\right.$ or $\left.\overline{R_{B}}(X)\right) \supseteq \overline{F R_{A+B}}(X)$.

Proof. It can be obtained easily by Propositions 31 and 33

Corollary 42. Let $\mathscr{I}=(U, A T, V, f)$ be an information system, $A_{i} \subseteq A T, i=1,2, \ldots, s$, and $X \subseteq U$. Then, the following properties hold.
(1) $\underline{S R_{\sum_{i=1}^{s} A_{i}}}(X) \subseteq \underline{R_{A_{i}}}(X) \subseteq \underline{F R_{\sum_{i=1}^{s} A_{i}}}(X) \subseteq \underline{R_{\cup_{i=1}^{s} A_{i}}}(X)$,
(2) $\overline{S R_{\sum_{i=1}^{s} A_{i}}}(X) \supseteq \underline{R_{A_{i}}}(X) \supseteq \overline{F R_{\sum_{i=1}^{s} A_{i}}}(X) \supseteq \underline{R_{\cup_{i=1}^{s} A_{i}}}(X)$.

Proposition 43. Let $\mathscr{I}=(U, A T, V, f)$ be an information system, $A_{i} \subseteq A T, i=1,2, \ldots, s$, and $X \subseteq U$. Then

$$
\rho_{\sum_{i=1}^{s} A_{i}}^{S}(X) \geq \rho_{A_{i}}(X) \geq \rho_{\sum_{i=1}^{s} A_{i}}^{F}(X) \geq \rho_{\sum_{i=1}^{s} A_{i}}(X) .
$$

Proof. It can be obtained directly by Definitions 8 and 21 and Corollary 42.

Proposition 44. Let $\mathscr{I}=(U, A T, V, f)$ be an information system, $A_{i} \subseteq A T, i=1,2, \ldots, s$, and $X \subseteq U$. Then

$$
\begin{aligned}
\gamma_{S}\left(\sum_{i=1}^{s} A_{i}, d\right) & \leq \gamma\left(A_{i}, d\right) \\
& \leq \gamma_{F}\left(\sum_{i=1}^{s} A_{i}, d\right) \leq \gamma\left(\bigcup_{i=1}^{s} A_{i}, d\right) .
\end{aligned}
$$

Proof. It can be obtained directly by Definitions 11 and 24 and Corollary 42.

One can understand the relationship and difference presented by the previous propositions though the following Figures 4 and 5. 


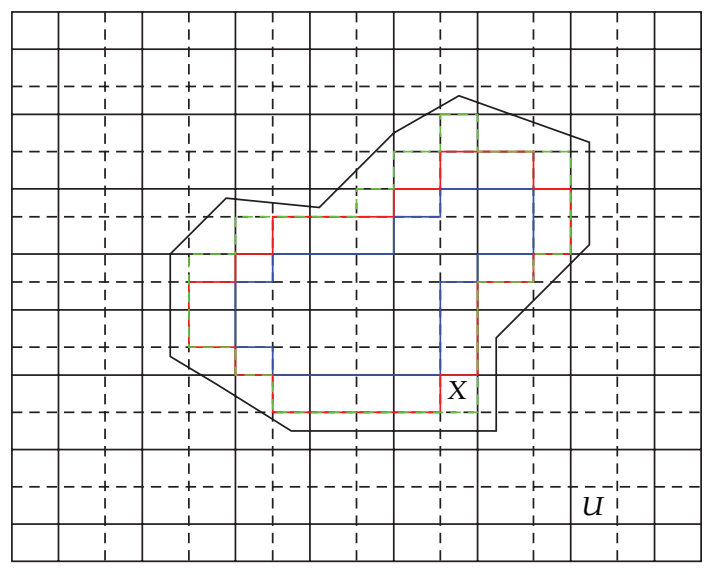

$$
-\frac{S R_{A+B}(X)}{F R_{A+B}(X)}-\begin{aligned}
& -R_{A} \\
& --R_{B}
\end{aligned}
$$

FIGURE 4: Difference and relationship of lower approximations among the 1st, the 2nd TGRS, and classical RS.

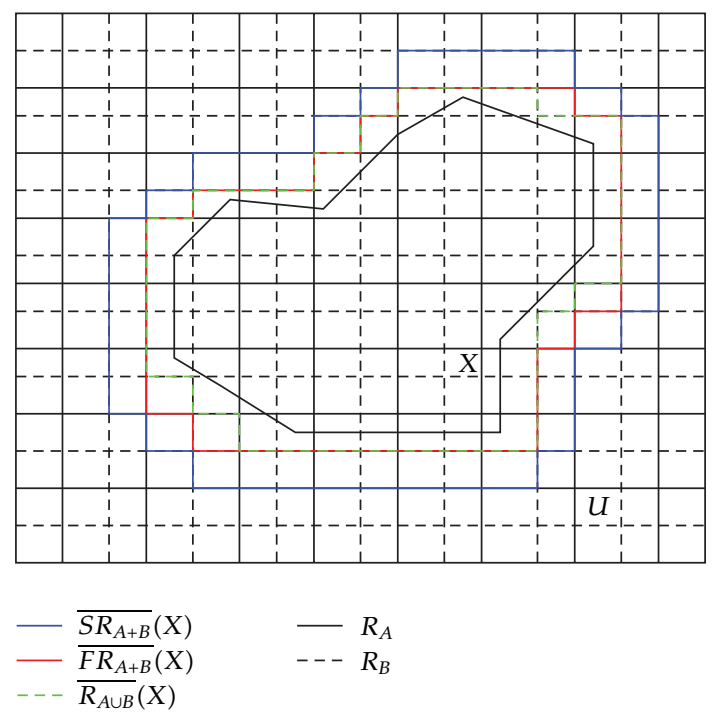

FIGURE 5: Difference and relationship of upper approximations among the 1st, the 2nd TGRS, and classical RS.

Example 45 (continued from Examples 4 and 17). In Examples 4 and 17, we have obtained that

$$
\begin{gathered}
\frac{R_{L \cup P}}{\overline{R_{L \cup P}}}(X)=\left\{u_{1}, u_{2}, u_{6}, u_{8}\right\}, \\
\left.\frac{F R_{L+P}}{}(X)=\left\{u_{2}, u_{6}, u_{8}\right\}, u_{2}, u_{8}\right\} \\
\overline{F R_{L+P}}(X)=\left\{u_{1}, u_{2}, u_{6}, u_{7}, u_{8}\right\} \\
\underline{S R_{L+P}}(X)=\left\{u_{2}\right\} \\
\overline{S R_{L+P}}(X)=\left\{u_{1}, u_{2}, u_{5}, u_{6}, u_{7}, u_{8}\right\}
\end{gathered}
$$

Obviously, we have

$$
\begin{aligned}
\frac{S R_{L+P}}{L}(X) & \subseteq \underline{F R_{L+P}}(X) \subseteq \underline{R_{L \cup P}}(X) \\
& \subseteq X \subseteq \overline{R_{L \cup P}}(X) \subseteq \overline{F R_{L+P}}(X) \subseteq \overline{S R_{L+P}}(X) .
\end{aligned}
$$

\section{Conclusion}

The original rough set model cannot be used to deal with the information systems with complicated context. Nevertheless, by relaxing the indiscernibility relation to more general binary relations, many improved rough set models have been successfully applied into the information systems with complicated context for knowledge acquisition. The contribution of this corresponding paper is to extend Pawlak's single granulation rough set model and Qian's multigranulation rough set model (MGRS) to two new types of the multiple granulation rough set model. In this paper, two new types of the multiple granulation rough set model have been constructed, respectively, based on multiple equivalence relations for an information system. In the two new types of multiple granulations rough set model, a target concept was approximated from two different kinds of views by using the equivalence classes induced from multiple granulations. In particular, some important properties of the two types of MGRS were investigated and have shown the relationship and difference among Pawlak's rough set, Qian's MGRS, and two new types of MGRS. Moreover, several important measures have been developed in two types of MGRS, such as rough measure and quality of approximation. From the contribution, it can be found that when two attribute sets in information systems possess a contradiction or an inconsistent relationship, or when efficient computation is required, the two new types of MGRS will display their advantage for rule extraction and knowledge discovery.

In our further research, we will extend other rough set methods in the context of multiple granulations such as viable precision rough set model and rough set induced by covering.

\section{Acknowledgments}

This work is supported by the Postdoctoral Science Foundation of China (no. 20100481331), the National Natural Science Foundation of Chongqing (no. cstc2011jjA40037) and the National Natural Science Foundation of China (no. 61105041, 71071124 and 11001227).

\section{References}

[1] Z. Pawlak, "Rough sets," International Journal of Computer and Information Sciences, vol. 11, no. 5, pp. 341-356, 1982.

[2] Z. Pawlak, Rough Set: Theoretical Aspects of Reasoning about Data, Kluwer Academic, Dordrecht, The Netherlands, 1991.

[3] Z. Pawlak and A. Skowron, "Rudiments of rough sets," Information Sciences, vol. 177, no. 1, pp. 3-27, 2007.

[4] R. W. Swiniarski and A. Skowron, "Rough set methods in feature selection and recognition," Pattern Recognition Letters, vol. 24, no. 6 , pp. 833-849, 2003. 
[5] S. Tsumoto, "Automated extraction of medical expert system rules from clinical databases based on rough set theory," Information Sciences, vol. 112, no. 1-4, pp. 67-84, 1998.

[6] V. S. Ananthanarayana, M. Narasimha Murty, and D. K. Subramanian, "Tree structure for efficient data mining using rough sets," Pattern Recognition Letters, vol. 24, no. 6, pp. 851-862, 2003.

[7] C.-C. Chan, "A rough set approach to attribute generalization in data mining," Information Sciences, vol. 107, no. 1-4, pp. 169-176, 1998.

[8] D. Kim, "Data classification based on tolerant rough set," Pattern Recognition, vol. 34, no. 8, pp. 1613-1624, 2001.

[9] Z. Pawlak, "Some remarks on conflict analysis," European Journal of Operational Research, vol. 166, no. 3, pp. 649-654, 2005.

[10] W. Cheng, Z. W. Mo, and J. Wang, "Notes on 'the lower and upper approximations in a fuzzy group' and 'rough ideals in semigroups,' Information Sciences, vol. 177, no. 22, pp. 51345140, 2007.

[11] B. Davvaz and M. Mahdavipour, "Roughness in modules," Information Sciences, vol. 176, no. 24, pp. 3658-3674, 2006.

[12] Q.-M. Xiao and Z.-L. Zhang, "Rough prime ideals and rough fuzzy prime ideals in semigroups," Information Sciences, vol. 176, no. 6, pp. 725-733, 2006.

[13] L. A. Zadeh, "Fuzzy logic equals computing with words," IEEE Transactions on Fuzzy Systems, vol. 4, no. 2, pp. 103-111, 1996.

[14] L. A. Zadeh, "Toward a theory of fuzzy information granulation and its centrality in human reasoning and fuzzy logic," Fuzzy Sets and Systems, vol. 90, no. 2, pp. 111-127, 1997.

[15] J. Y. Liang and D. Y. Li, Uncertainty and Knowledge Acquisition in Information Systems, Science Press, Beijing, China, 2005.

[16] Q. H. Hu, D. R. Yu, and Z. X. Xie, "Reduction algorithms for hybrid data based on fuzzy rough set approaches," in Proceedings of the International Conference on Machine Learning and Cybernetics, pp. 1469-1474, August 2004.

[17] D. Li, B. Zhang, and Y. Leung, "On knowledge reduction in inconsistent decision information systems," International Journal of Uncertainty, Fuzziness and Knowledge-Based Systems, vol. 12, no. 5, pp. 651-672, 2004.

[18] W. X. Zhang, J. S. Mi, and W. Z. Wu, "Approaches to knowledge reductions in inconsistent systems," International Journal of Intelligent Systems, vol. 18, no. 9, pp. 989-1000, 2003.

[19] W. X. Zhang, W. Z. Wu, J. Y. Liang, and D. Y. Li, Theory and Method of Rough Sets, Science Press, Beijing, China, 2001.

[20] B. Apolloni, A. Brega, D. Malchiodi, G. Palmas, and A. M. Zanaboni, "Learning rule representations from data," IEEE Transactions on Systems, Man, and Cybernetics A, vol. 36, no. 5, pp. 1010-1028, 2006.

[21] T. P. Hong, L. H. Tseng, and S. L. Wang, "Learning rules from incomplete training examples by rough sets," Expert Systems with Applications, vol. 22, no. 4, pp. 285-293, 2002.

[22] S. Tsumoto, "Automated extraction of hierarchical decision rules from clinical databases using rough set model," Expert Systems with Applications, vol. 24, no. 2, pp. 189-197, 2003.

[23] N. Zhong, J. Dong, and S. Ohsuga, "Rule discovery by soft induction techniques," Neurocomputing, vol. 36, no. 1-4, pp. 171204, 2001.

[24] J. Liang and Y. Qian, "Information granules and entropy theory in information systems," Science in China F, vol. 51, no. 10, pp. 1427-1444, 2008.
[25] Z. Pawlak, "Rough sets, decision algorithms and Bayes' theorem," European Journal of Operational Research, vol. 136, no. 1, pp. 181-189, 2002.

[26] A. Bargiela and W. Pedrycz, "Granular mappings," IEEE Transactions on Systems, Man, and Cybernetics A, vol. 35, no. 2, pp. 292-297, 2005.

[27] T. Y. Lin, "From rough sets and neighborhood systems to information granulation and computing in words," in Proceedings of the 2nd European Congress on Intelligent Techniques and Soft Computing, pp. 602-1606, September 1997.

[28] W. Pedrycz, "Relational and directional aspects in the construction of information granules," IEEE Transactions on Systems, Man, and Cybernetics A, vol. 32, no. 5, pp. 605-614, 2002.

[29] W. Pedrycz and A. Bargiela, "Granular clustering: a granular signature of data," IEEE Transactions on Systems, Man, and Cybernetics B, vol. 32, no. 2, pp. 212-224, 2002.

[30] Y. Y. Yao, "Information granulation and rough set approximation," International Journal of Intelliegent Systmes, vol. 16, no. 1, pp. 87-104, 2001.

[31] L. A. Zadeh, "A new direction in AI: toward a computational theory of perceptions," AI Magazine, vol. 22, no. 1, pp. 73-84, 2001.

[32] A. Skowron and J. Stepaniuk, "Tolerance approximation spaces," Fundamenta Informaticae, vol. 27, no. 2-3, pp. 245-253, 1996.

[33] Y. Y. Yao, "Relational interpretations of neighborhood operators and rough set approximation operators," Information Sciences, vol. 111, no. 1-4, pp. 239-259, 1998.

[34] D. Ślęzak and W. Ziarko, "The investigation of the Bayesian rough set model," International Journal of Approximate Reasoning, vol. 40, no. 1-2, pp. 81-91, 2005.

[35] D. Dbois and H. Prade, "Rough fuzzy sets and fuzzy rough sets," International Journal of General Systems, vol. 17, no. 2-3, pp. 191209, 1990.

[36] Q. Hu, D. Yu, Z. Xie, and J. Liu, "Fuzzy probabilistic approximation spaces and their information measures," IEEE Transactions on Fuzzy Systems, vol. 14, no. 2, pp. 191-201, 2006.

[37] Y. Leung, W.-Z. Wu, and W.-X. Zhang, "Knowledge acquisition in incomplete information systems: a rough set approach," European Journal of Operational Research, vol. 168, no. 1, pp. 164-180, 2006.

[38] Z. Pawlak and A. Skowron, "Rough sets: some extensions," Information Sciences, vol. 177, no. 1, pp. 28-40, 2007.

[39] K. Thangavel and A. Pethalakshmi, "Dimensionality reduction based on rough set theory: a review," Applied Soft Computing Journal, vol. 9, no. 1, pp. 1-12, 2009.

[40] W.-Z. Wu, Y. Leung, and W.-X. Zhang, "Connections between rough set theory and Dempster-Shafer theory of evidence," International Journal of General Systems, vol. 31, no. 4, pp. 405430, 2002.

[41] W.-Z. Wu, M. Zhang, H.-Z. Li, and J.-S. Mi, "Knowledge reduction in random information systems via Dempster-Shafer theory of evidence," Information Sciences, vol. 174, no. 3-4, pp. 143-164, 2005.

[42] W.-H. Xu and W.-X. Zhang, "Measuring roughness of generalized rough sets induced by a covering," Fuzzy Sets and Systems, vol. 158, no. 22, pp. 2443-2455, 2007.

[43] W. H. Xu, X. Y. Zhang, and W. X. Zhang, "Knowledge granulation, knowledge entropy and knowledge uncertainty measure in ordered information systems," Applied Soft Computing Journal, vol. 9, no. 4, pp. 1244-1251, 2009. 
[44] W. H. Xu, J. M. Zhong, X. Y. Zhang, and W. X. Zhang, "Attribute reduction in ordered information systems based on evidence theory," Knowledge and Information Systems, vol. 25, no. 1, pp. 169-184, 2010.

[45] D. Yu, Q. Hu, and C. Wu, "Uncertainty measures for fuzzy relations and their applications," Applied Soft Computing Journal, vol. 7, no. 3, pp. 1135-1143, 2007.

[46] L. A. Zadeh, "Fuzzy Sets and Information Granularity," in Advances in Fuzzy Set Theory and Applications, pp. 3-18, North Holland, Amsterdam, The Netherlands, 1979.

[47] Z. Pawlak and R. Sowinski, "Rough set approach to multiattribute decision analysis," European Journal of Operational Research, vol. 72, no. 3, pp. 443-459, 1994.

[48] L. A. Zadeh, "Fuzzy sets," Information and Computation, vol. 8, pp. 338-353, 1985.

[49] G. Shafer, A Mathematical Theory of Evidence, Princeton University Press, Princeton, NJ, USA, 1976.

[50] J. R. Hobbs, "Granularity," in Proceedings of the 9th International Joint Conference on Artificial Intelligence (IJCAI '85), pp. 432435, Los Angeles, Calif, USA, 1985.

[51] G. J. Klir, "Basic issues of computing with granular probabilities," in Proceedings of the IEEE International Conference on Fuzzy Systems, pp. 101-105, May 1998.

[52] J. Y. Liang and Y. H. Qian, "Axiomatic approach of knowledge granulation in information system," in AI 2006: Advances in Artificial Intelligence, A. Sattar and B. H. Kang, Eds., Lecture Notes in Computer Science, pp. 1074-1078, Springer, Berlin, Germany, 2006.

[53] J. Liang, Z. Shi, D. Li, and M. J. Wierman, "Information entropy, rough entropy and knowledge granulation in incomplete information systems," International Journal of General Systems, vol. 35, no. 6, pp. 641-654, 2006.

[54] T. Y. Lin, "Introduction to special issues on data mining and granular computing," International Journal of Approximate Reasoning, vol. 40, no. 1-2, pp. 1-2, 2005.

[55] Y. Qian and J. Liang, "Combination entropy and combination granulation in rough set theory," International Journal of Uncertainty, Fuzziness and Knowledge-Based Systems, vol. 16, no. 2, pp. 179-193, 2008.

[56] Y. Y. Yao, "Granular computing basis issues and possible solutions," in Proceedings of the 5th International Conference Computing and Information, pp. 186-189, 2000.

[57] Y. Y. Yao, "A partition model of granular computing," LNCS Transactions on Rough Sets I, vol. 3100, pp. 232-253, 2004.

[58] Y. Y. Yao, "Three perspectives of granular computing, The Proceedings, International Forum on Theory of GrC from Rough Set Perspective," Journal of Nanchang Institute of Technology, vol. 25, no. 2, pp. 16-21, 2006.

[59] Y. Yao, "Perspectives of granular computing," in Proceedings of the IEEE International Conference on Granular Computing, pp. 85-90, July 2005.

[60] Y. Qian, J. Liang, Y. Yao, and C. Dang, "MGRS: a multigranulation rough set," Information Sciences, vol. 180, no. 6, pp. 949-970, 2010. 


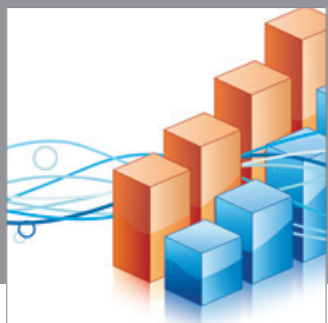

Advances in

Operations Research

mansans

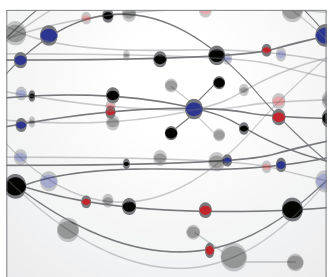

The Scientific World Journal
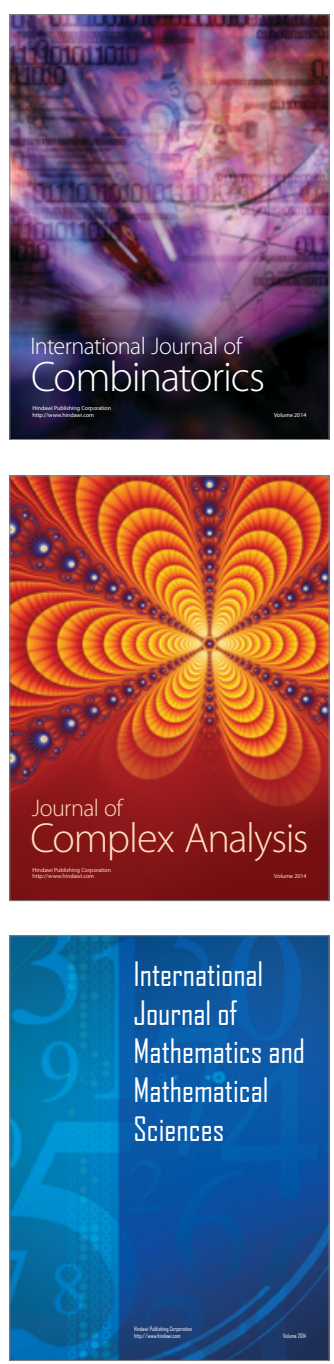
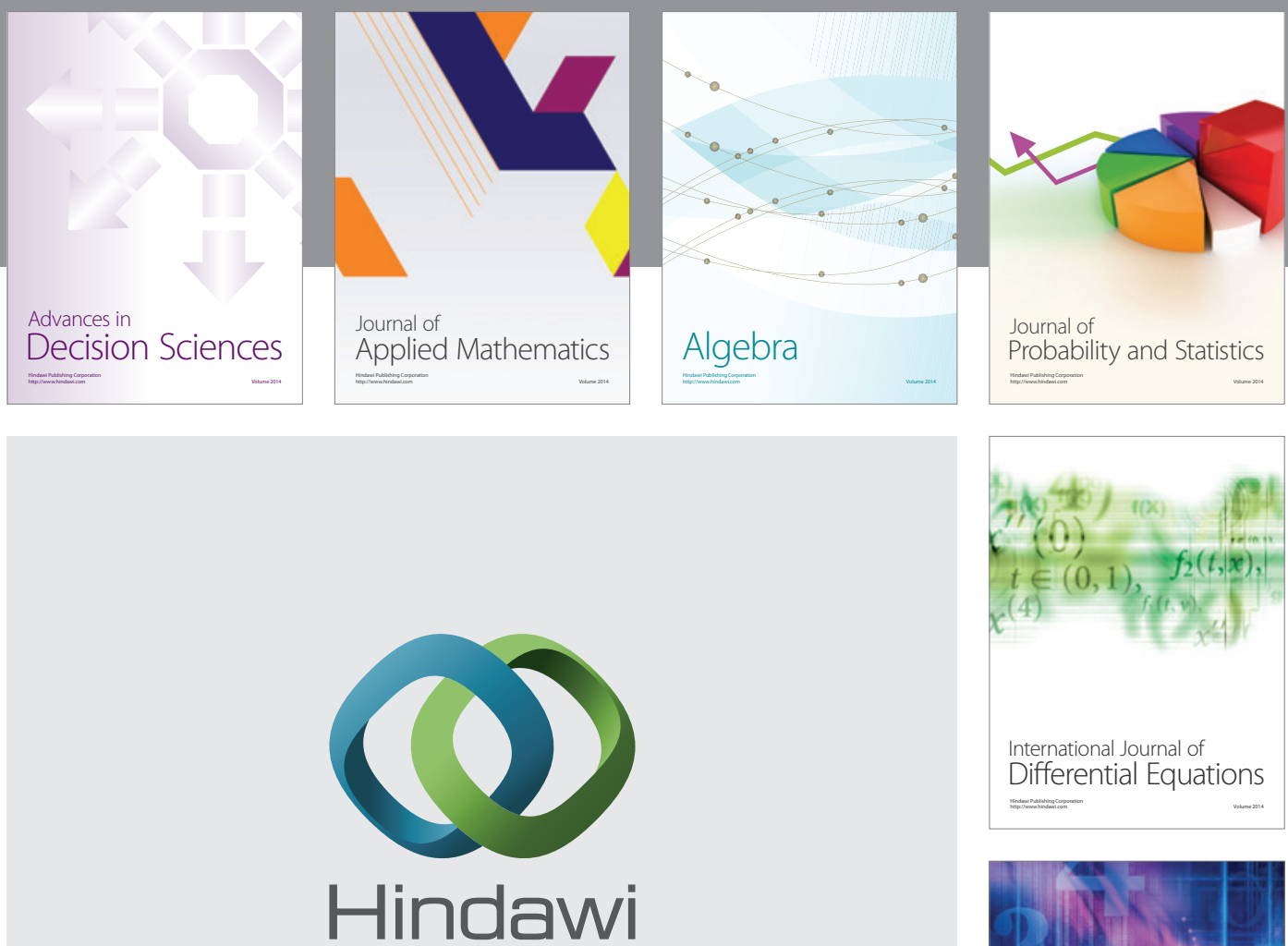

Submit your manuscripts at http://www.hindawi.com
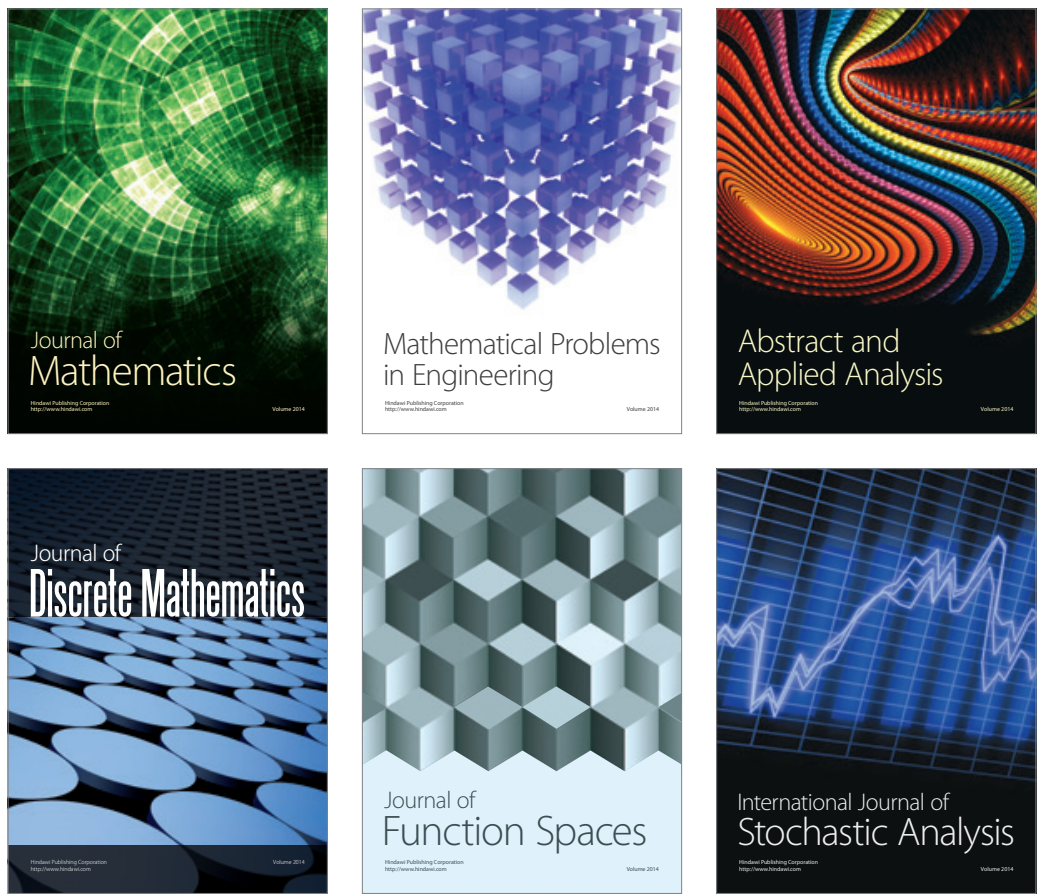

Journal of

Function Spaces

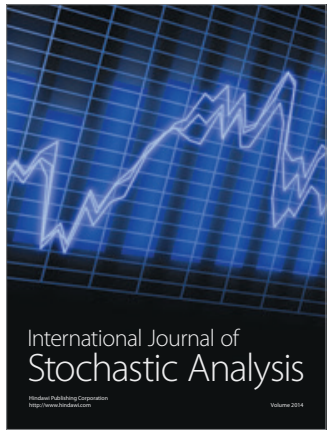

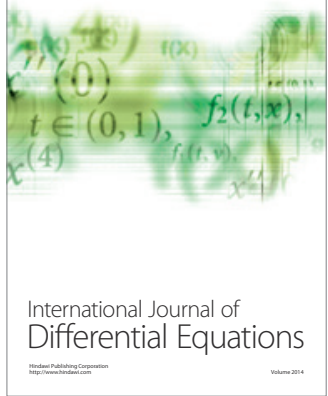
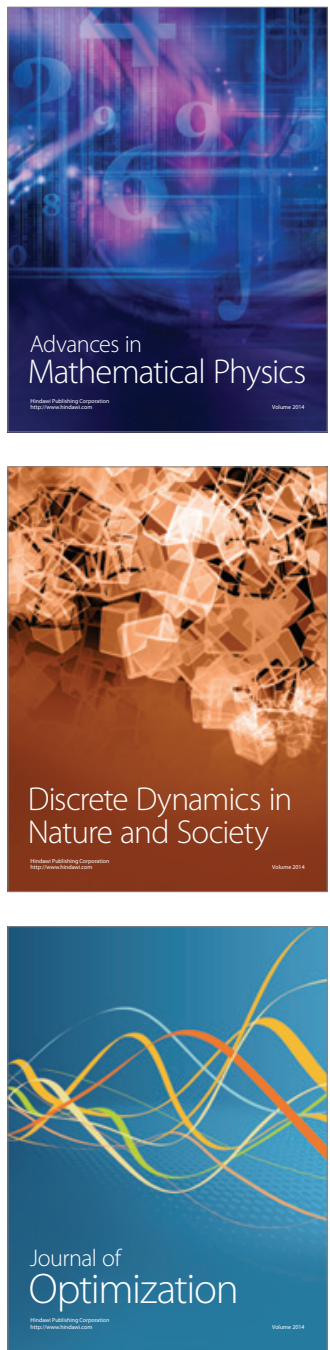